\title{
Altered Proinflammatory Responses to Polyelectrolyte Multilayer Coatings Are Associated with Differences in Protein Adsorption and Wettability
}

Florian Billing,* Bernadette Walter, Simon Fink, Elsa Arefaine, Luisa Pickarski, Sandra Maier, Robin Kretz, Meike Jakobi, Nora Feuerer, Nicole Schneiderhan-Marra, Claus Burkhardt, Markus Templin, Anne Zeck, Rumen Krastev, Hanna Hartmann, and Christopher Shipp

Cite This: ACS Appl. Mater. Interfaces 2021, 13, 55534-55549 Read Online

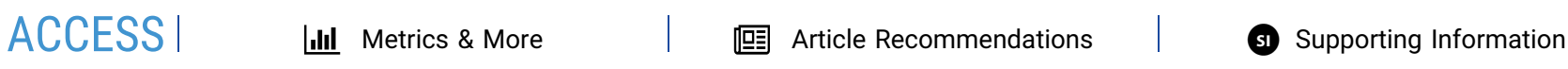

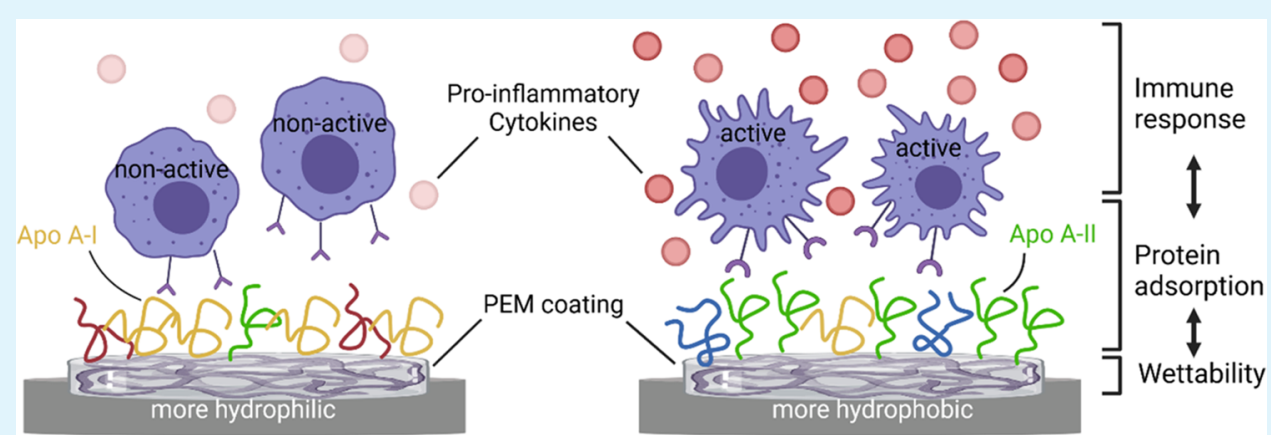

ABSTRACT: A full understanding of the relationship between surface properties, protein adsorption, and immune responses is lacking but is of great interest for the design of biomaterials with desired biological profiles. In this study, polyelectrolyte multilayer (PEM) coatings with gradient changes in surface wettability were developed to shed light on how this impacts protein adsorption and immune response in the context of material biocompatibility. The analysis of immune responses by peripheral blood mononuclear cells to PEM coatings revealed an increased expression of proinflammatory cytokines tumor necrosis factor (TNF)- $\alpha$, macrophage inflammatory protein (MIP)- $1 \beta$, monocyte chemoattractant protein (MCP)-1, and interleukin (IL)- 6 and the surface marker CD86 in response to the most hydrophobic coating, whereas the most hydrophilic coating resulted in a comparatively mild immune response. These findings were subsequently confirmed in a cohort of 24 donors. Cytokines were produced predominantly by monocytes with a peak after $24 \mathrm{~h}$. Experiments conducted in the absence of serum indicated a contributing role of the adsorbed protein layer in the observed immune response. Mass spectrometry analysis revealed distinct protein adsorption patterns, with more inflammation-related proteins (e.g., apolipoprotein A-II) present on the most hydrophobic PEM surface, while the most abundant protein on the hydrophilic PEM (apolipoprotein A-I) was related to anti-inflammatory roles. The pathway analysis revealed alterations in the mitogen-activated protein kinase (MAPK)-signaling pathway between the most hydrophilic and the most hydrophobic coating. The results show that the acute proinflammatory response to the more hydrophobic PEM surface is associated with the adsorption of inflammation-related proteins. Thus, this study provides insights into the interplay between material wettability, protein adsorption, and inflammatory response and may act as a basis for the rational design of biomaterials.

KEYWORDS: protein adsorption, polyelectrolyte multilayers, wettability, immune response, PBMCs

\section{INTRODUCTION}

The inflammatory reaction of the host is crucial for implant biocompatibility. ${ }^{1}$ Hereby, the type of response by the recruited immune cells is a decisive factor in whether a medical implant will successfully perform its intended function or whether it results in detrimental immune responses contributing to fibrosis, chronic inflammation, or other unwanted effects. ${ }^{2}$ To actively modulate the immune response, the implant design has moved into focus ${ }^{3}$ as physicochemical surface properties of the applied biomaterials are found to impact the outcome of the provoked immune response. ${ }^{4-8}$ For example, certain degrees of wettability or surface charge are associated with positive immunological effects, ${ }^{8,9}$ although material-specific differences should not be neglected. However,

Received: August 24, 2021

Accepted: October 27, 2021

Published: November 11, 2021 
surface properties affect the immune response only in an indirect manner.

A decisive mediating role between the biomaterial and immune cells is performed by the proteins adsorbed on the biomaterial surface. Within seconds after the first materialtissue contact, proteins from blood and interstitial fluids begin adsorbing onto the biomaterial, thus providing the surface with a new biological identity. ${ }^{10,11}$ Therefore, subsequently arriving immune cells mostly sense foreign surfaces through this adsorbed layer but not the surface itself. The adsorbed serum proteins can influence the behavior of immune cells, which directly affect the immunological outcome of a biomaterial. ${ }^{11-16}$ In this context, biomaterial surface parameters such as wettability and surface charge are thought to have a significant impact on the nature, volume, and conformation of adsorbed proteins. ${ }^{10,11,14,16,17}$ Hence, cellular responses to a biomaterial in a biological medium seem to be dependent on the adsorbed biomolecule layer, which is built up based on the material's physicochemical properties. However, there remains a paucity of data on interrelations between these three decisive factors determining the fate of a biomaterial implant. While previous in vitro studies mainly focused on either the correlation between surface properties and protein adsorption ${ }^{17,18}$ or the modulation of the immune response by artificial protein coatings, ${ }^{12,15,19}$ only a few included all three aspects. Moreover, previous investigations often relied on simplified models. Thus, a thorough investigation that considers the complexities of each factor is lacking. In this study, we attempt to represent the complex nature of surface-protein interactions as well as the full spectrum of the immune response, while pinpointing the effects to specific differences in material properties.

The choice of an appropriate biomaterial surface is crucial for the identification of surface parameters being specifically responsible for the observed immune response. Hereby, a precise assignment is difficult when comparing materials with different chemical compositions and a variety in several surface parameters. However, this knowledge is important for the targeted design of immunocompatible implants. ${ }^{4,16}$ To link particular modulations to specific immunological outcomes, we employed polyelectrolyte multilayer (PEM) coatings. On a chemical level, PEMs are nanometer-scale polymer films that are generated by alternating deposition of polyelectrolytes utilizing layer-by-layer (LbL) self-assembly. ${ }^{20}$ Due to their versatile nature, PEM coatings can be deposited on a wide range of substrates including medical devices. They have attracted attention in the field of biomedical engineering as they may be utilized to control protein adsorption, promote cell adhesion, and modulate the inflammatory response, ${ }^{21}$ though there are still relatively few publications on the biological aspects of this technology. Modifications of certain deposition parameters, such as $\mathrm{pH}$ conditions, salts, temperature, etc. can be applied to impart changes in the physicochemical properties like surface charge or wettability. $^{22,23}$ For this study, we aimed for the application of a set of PEM coatings based on identical polymers that vary only in a small subset of physicochemical properties.

The complexity of the immunological model applied is another critical factor for developing a comprehensive understanding of the interrelation between surface parameters and the immune response. Here, previous investigations often rely on a simplified model of the immune system, using single populations of immune cells such as macrophage cell lines. ${ }^{14}$ However in vivo, also other types of immune cells such as monocytes and lymphocyte populations are found in the biomaterial surrounding tissues (particularly in cases of aseptic loosening and implant failure), thus implicating a contribution of these cell types to the observed immune response. ${ }^{24,25}$ The inclusion of all cell populations involved is therefore essential for an accurate in vitro analysis of the immune response and enables the assessment of immune-relevant cell types in addition. To investigate the influence of certain surface alterations on the immune system, we employed a humanbased in vitro immune model consisting of primary peripheral blood mononuclear cells (PBMCs). These cells encompass almost the entire spectrum of circulating immune cells, including innate immune cells like monocytes and natural killer cells as well as helper and cytotoxic $T$ cells of the adaptive immune system, among other less frequent populations. This broad immune cell spectrum resembles the in vivo situation quite closely and their patient-derived nature makes them suitable for assessing biological differences across individuals.

The use of a complex model is also critical in determining the contribution of adsorbed proteins. Many previous studies were conducted by analyzing the effect of single proteins or simple mixtures of selected proteins, e.g., albumin, fibronectin/ fibrinogen, and immunoglobulin. ${ }^{12,19,26,27}$ However, implanted devices are confronted with blood plasma containing thousands of protein compounds and the entire spectrum of extracellular matrix proteins. As a result, simplified models often fail to account for the complex and dynamic adsorption patterns in vivo. ${ }^{28}$ Therefore, in vitro testing with human serum is likely more representative of protein adsorption in vivo. ${ }^{29}$ Thus, in this study, human serum was included in the cell culture medium in all experiments. To identify relevant protein compounds, mass spectrometry was employed to quantify serum-derived protein adsorption. By comparing the protein composition on the different PEM coatings, we furthermore aimed to correlate the adsorbed protein layer with the observed inflammatory response. In the last step, protein expression profiling was applied using DigiWest analysis to identify alterations in cellular signal transduction in response to the distinct PEM coatings. By analyzing the adsorbed serum proteins and the potential signaling pathways, we aimed to develop a deeper understanding of the relationship between material surface properties, protein adsorption, and the resulting immune response.

\section{METHODS}

2.1. Material Characterization. 2.1.1. Polyelectrolyte Solutions. For the buildup of PEMs, sodium poly(styrene sulfonate) (PSS; $M_{\mathrm{w}}$ $70 \mathrm{kDa}$ ) and polyethyleneimine (PEI; $M_{\mathrm{w}} 750 \mathrm{kDa}$ ) were both obtained from Sigma-Aldrich (Steinheim, Germany). Poly(allylamine hydrochloride) (PAH) was purchased from Alfa Aesar (Haverhill, Massachusetts). The polymers were used without further purification. Sodium chloride and sterile, pyrogen-free water were obtained from Carl Roth (Karlsruhe, Germany). The concentration of PSS and PAH was $2 \mathrm{mg} / \mathrm{mL}$ in $0.5 \mathrm{mM}$ sodium chloride at $\mathrm{pH}$ values 4,7 , and 9 .

2.1.2. Manufacture of Polyelectrolyte Multilayer (PEM) Coatings. The polycationic PEMs PEI(PSS/PAH $)_{5}$ were prepared manually at room temperature $(\mathrm{RT})$ using the layer-by-layer ( $\mathrm{LbL}$ ) technique. The film buildup was prepared under sterile conditions. First, a layer of PEI $(10 \mathrm{mg} / \mathrm{mL}, \mathrm{pH} 7)$ was deposited for $10 \mathrm{~min}$. After incubation, the samples were extensively rinsed with sterile, pyrogen-free water (3 $\times 2 \mathrm{~min}$ ). Next, five bilayers of PSS/PAH were deposited by alternate application (i.e., $10 \mathrm{~min}$ PSS and PAH solutions) with an intermediate washing step, which altogether lasted over $6 \mathrm{~min}$ in water. Three different types of coatings using the identical PSS/PAH substrates were generated by adjusting the $\mathrm{pH}$ of the assembling 
solutions during the creation process to $\mathrm{pH}$ values of 4,7 , and 9, which are referred to as cationic $\mathrm{A}, \mathrm{B}$, and $\mathrm{C}$ in this manuscript, respectively. Notably, the $\mathrm{pH}$ state of the assembling solution of the PEMs has no influence on the $\mathrm{pH}$ value in the subsequent cell culture experiments. For all experiments involving PBMCs, coatings were applied directly onto 24-well tissue culture plates (TCP, Sarstedt, Nürnbrecht, Germany), resulting in a surface area of $1.82 \mathrm{~cm}^{2}$ per sample.

2.1.3. Ellipsometry. The film growth was monitored by means of an SENpro (SENTECH Instruments $\mathrm{GmbH}$, Germany) spectroscopic ellipsometer with a wavelength range from 429 to $900 \mathrm{~nm}$, at an angle of incidence of $70^{\circ}$. To obtain the film thickness, the raw data were fitted with a four-layer model considering the contribution from the air, $\mathrm{PEM}, \mathrm{SiO}_{2}$, and $\mathrm{Si}$.

2.1.4. $\zeta$ Potential. The $\zeta$ potential was determined on PEM coatings (PerkinElmer Inc.) using a Zetasizer Nano ZS particle analyzer (Malvern Instruments, Herrenberg, Germany) at $25^{\circ} \mathrm{C}$ and a $173^{\circ}$ scattering angle by cumulative analysis. $\zeta$ potential analysis based on the electrophoretic mobility of the nanoplexes in aqueous buffer was performed using folded capillary cells (Malvern Instruments, Herrenberg, Germany) in automatic mode and calculated using the Smoluchowski equation. $\zeta$ potential measurements were done in triplicate and reproduced three times $(n=3$ experiments and $n=9$ total measurements).

2.1.5. Water Contact Angle. The wettability of each sample was determined by sessile drop measurements of the water contact angle $(\theta)$ using a Krüss Easy Drop contact angle goniometer (Hamburg, Germany). To perform the measurements, a static drop was used, maintaining a constant volume during the measurement. This was performed with the addition of $2 \mu \mathrm{L}$ of distilled water to the samples. After $20 \mathrm{~s}$, an image was recorded and transferred to the software for analysis of the static (equilibrium) contact angles. Three independent experiments were performed in triplicate to give the mean value \pm standard deviation (SD).

2.1.6. Bovine Serum Albumin (BSA) Adsorption. For quantification of the protein adsorption, a solution of BSA was used. The concentration was $40 \mathrm{mg} / \mathrm{mL}$, corresponding to the physiological concentration of human serum albumin in blood. ${ }^{30}$ The lyophilized protein was dissolved in modified simulated body fluid (m-SBF) with ion concentrations, corresponding to those in human blood plasma. ${ }^{31}$ The used BSA was fluorescently labeled to allow quantification of the resulting protein adsorption and was applied as a $4 \%$ additive in the used BSA-fluorescein isothiocyanate (FITC) solution. Coated well plates were washed $3 \times$ with $\mathrm{m}-\mathrm{SBF}$, incubated for 20 min with BSAFITC in $\mathrm{m}-\mathrm{SBF}$, and washed again $3 \times$ with $\mathrm{m}-\mathrm{SBF}$ before measurement. The fluorescence intensity was quantified using a PHERAstar microplate reader (BMG Labtech). Empty wells (blank), $\mathrm{m}-\mathrm{SBF}$, and BSA-FITC alone served as controls.

2.1.7. Scanning Electron Microscopy (SEM). PBMCs for SEM analysis were cultured in 24-well cell culture plates coated with PEM cationic $\mathrm{A}, \mathrm{B}$, and $\mathrm{C}$ for $24 \mathrm{~h}$ at $37{ }^{\circ} \mathrm{C}$ and $5 \% \mathrm{CO}_{2}$ in a humidified atmosphere. After culture, cells were washed three times with phosphate-buffered saline (PBS) (Thermo Fisher Scientific) and fixed for $2 \mathrm{~h}$ on ice with a PBS solution containing $4 \%$ paraformaldehyde (PFA) and $2 \%$ glutaraldehyde (both w/v). Subsequently, samples were washed three times with PBS. Dehydration was performed using a graded series of ethanol solutions (30, 50, 70, 80, 95, 100\%). Following critical point drying (Baltec CPD 030), samples were coated with $5 \mathrm{~nm}$ gold-palladium (Cressington $208 \mathrm{HR}$ ). Surface images were taken with an Auriga $40 \mathrm{FIB} / \mathrm{SEM}$ (Zeiss, Oberkochen, Germany) at a $3 \mathrm{keV}$ acceleration voltage using a chamber secondary electron detector.

2.2. Isolation of PBMCs from Whole Blood. Whole blood samples of healthy volunteers were taken between November 2018 and October 2020 after written informed consent. Potential donors were screened with exclusion criteria (chronic inflammatory disease, surgical intervention within the last 3 months, infection or use of medications affecting the immune system in the past 2 weeks, vaccination in the previous 6 weeks, excess alcohol consumption, or strenuous exercise prior to donation) to minimize the influence by environmental factors known to alter the immune system. All blood draws took place in the morning hours (9-11 am) to ensure consistency. Immediately after the blood was drawn, PBMCs were isolated by density gradient centrifugation using SepMate isolation tubes (StemCell Technologies, Cologne, Germany) according to the manufacturer's protocol. Isolated PBMCs were stored at $-150^{\circ} \mathrm{C}$ in a medium containing 10\% dimethyl sulfoxide (DMSO) (Sigma-Aldrich, Hamburg, Germany), 20\% fetal calf serum (FCS) (Sigma-Aldrich), and 70\% Roswell Park Memorial Institute Medium (RPMI) 1640 (Gibco, Paisley, Scotland) until use. As shown in a previous publication, ${ }^{32}$ the freezing/thawing procedure did not affect the cellular activity of the cells. The viability of the PBMCs was assessed prior to each experiment by the trypan blue exclusion assay and was found to be greater than $97 \%$ in all cases.

2.3. PBMC Cell Culture. Unless otherwise stated, PBMCs were cultured in Iscove's modified Dulbecco's medium (IMDM medium, Gibco, Thermo Fisher Scientific) containing GlutaMAX supplemented with $10 \%$ off-the-clot serum pooled from male $\mathrm{AB}$ blood group donors (human $\mathrm{AB}$ pooled serum (male), H2B, Limoges, France). In experiments assessing the general role of serum proteins, PBMCs were cultured in X-VIVO 15 serum-free medium (Lonza, Basel, Switzerland). In these cases, prior to culturing of the cells, the surfaces were either incubated with human serum $(\mathrm{H} 2 \mathrm{~B})$ at $37{ }^{\circ} \mathrm{C}$ for $1 \mathrm{~h}$ and washed $5 \times$ with PBS, or remained untreated. For all experiments, $5.0 \times 10^{5}$ cells were seeded in $500 \mu \mathrm{L}$ of medium. Cells stimulated with PHA-L $(10 \mu \mathrm{g} / \mathrm{mL}$, Roche, Mannheim, Germany) and lipopolysaccharides (LPSs) from Escherichia coli O55:B55 (100 $\mathrm{ng} / \mathrm{mL}$, Hycult Biotech, Uden, the Netherlands) served as a proinflammatory positive control to ensure cell functionality. PBMCs cultured on uncoated tissue culture plates (TCPs) without treatment were used as the negative control. Depending on the specific experiment, culture periods were $12,24,72$, or $96 \mathrm{~h}$ at $37^{\circ} \mathrm{C}$ and $5 \% \mathrm{CO}_{2}$ in a humidified atmosphere. Throughout all culturing periods, the medium was not exchanged, which allowed nonadherent cells to remain in the culture and to potentially interact with adherent cells via contact-independent mechanisms. For experiments employing intracellular cytokine staining, $1 \times$ Brefeldin A (BioLegend, Amsterdam, the Netherlands) was applied to the culture $12 \mathrm{~h}$ prior to harvesting to prevent cytokine secretion. In the case of experiments assessing secreted cytokines, multiplex (Luminex) immunoassays were employed. For this, $190 \mu \mathrm{L}$ of cell culture medium was collected following the culture period and centrifuged at $5000 \mathrm{rpm}$ for $3 \mathrm{~min}$. The supernatant was then collected and stored at $-80{ }^{\circ} \mathrm{C}$ until analysis.

2.4. Cytokine Analysis Using Multiplexed Bead-Based Sandwich Immunoassays. To analyze concentrations of chemoand cytokines, collected supernatants were thawed and spun down at $10000 \mathrm{~g}$ for $5 \mathrm{~min}$ to remove remaining cells. Levels of interleukin (IL)-1 $\beta$, IL-1RA, IL-4, IL-6, IL-8, IL-10, IL-12p70, IL-13, GM-CSF, interferon (IFN) $-\gamma$, monocyte chemoattractant protein (MCP)-1, macrophage inflammatory protein (MIP)- $1 \beta$, tumor necrosis factor (TNF)- $\alpha$, and vascular endothelial growth factor (VEGF) were quantified using a set of Luminex-based sandwich immunoassays, which had been developed in-house and described previously. ${ }^{33}$ Each assay consisted of commercially available capture and detection antibodies and calibrator proteins. Assays had been thoroughly validated ahead of the study with respect to accuracy, precision, parallelism, robustness, specificity, and sensitivity. ${ }^{34,35}$ The dilution factor of the samples was at least 1:4 or higher. After incubation of the prediluted samples or calibrator protein with the capture coated microspheres, beads were washed and incubated with biotinylated detection antibodies. Streptavidin-phycoerythrin (PE) was added after an additional washing step for visualization. As a control, calibrators and quality control samples were also tested on each microtiter plate. Plates were measured using a Luminex FlexMap 3D analyzer system with Luminex xPONENT 4.2 software (Luminex, Austin). Data was analyzed with MasterPlex QT version 5.0. Evaluation of the standard curve and quality control samples was performed in accordance with internal criteria adapted to the Westgard rules to guarantee the proper performance of assays. ${ }^{36}$ 
2.5. Flow Cytometry. Following the culture period, cells were harvested, as described previously. ${ }^{32}$ Staining with extracellular antibodies was performed for $30 \mathrm{~min}$ on ice using the following antibodies: CD3-PerCP Cy5.5 (\#317336), CD4-Alexa488 (\#317420), CD16-BV605 (\#302040), CD25-PE-Cy5 (\#302607), CD86-BV650 (\#305428), CD127-BV711 (\#351328), CD163-PE/dazzle594 (\#333624), CD206-PE/Cy7 (\#321124), CD284-APC (\#312815), and HLA-DR-BV421 (\#307636), all obtained from Biolegend, and CD14-APC-H7 (\#560270) and CD354-BV510 (\#743739), both from BD Biosciences (Heidelberg, Germany). For the mixed surface marker/intracellular staining panel, the cells were stained with CD3APC (\#317317), CD14-PerCP/Cy5.5 (\#367109), and CD16-BV605 (\#302039), all obtained from Biolegend. After staining, cells were washed, spun, and the supernatant was discarded. For intracellular staining, cells were permeabilized using a Cytofix/Cytoperm Fixation/ Permeabilization kit (BD) in accordance with the manufacturer's instructions before intracellular proteins were blocked for $10 \mathrm{~min}$ with $10 \%(\mathrm{v} / \mathrm{v})$ human serum diluted in permeabilization buffer. After washing, intracellular cytokines were labeled using IL-6-PE/ Dazzle594 (\#501122), IL-8-Alexa Fluor488 (\#511412), TNF- $\alpha$ BV711 (\#502940), and MCP-1-PE/Cy7 (\#502614) (all Biolegend) and MIP- $1 \beta$-BV421 (BD) antibodies diluted in permeabilization buffer and incubated for $30 \mathrm{~min}$ on ice in the dark. After the last washing step, stained cells were immediately analyzed using an LSRFortessa instrument with the FACSDiva software version 8.0.3 (BD). All washing steps were performed with $1 \mathrm{~mL}$ of washing buffer and centrifugation at $300 \mathrm{~g}$ for $5 \mathrm{~min}$. Data analysis was performed using FlowJo software v10.5 (BD) and analyzed according to the gating strategy shown in Figures S1 and S2.

2.6. On-Surface Trypsin Digestion and Liquid Chromatography-Mass Spectrometry (LC-MS/MS) Analysis. PEM coatings were incubated with $10 \%$ HS in IMDM medium without cells for $1 \mathrm{~h}$ at $37{ }^{\circ} \mathrm{C}$. The surfaces were washed $5 \times$ with PBS and afterward incubated overnight with $5 \mu \mathrm{g}$ of trypsin (Worthington) in $50 \mathrm{mM}$ tris (Carl Roth), pH 7.5 at $37^{\circ} \mathrm{C}$. The proteolysis was stopped by adding formic acid (99\% LC-MS grade from Fisher Chemicals) to a final concentration of $7 \%(\mathrm{v} / \mathrm{v})$. Tryptic peptides were analyzed by liquid chromatography-mass spectrometry (LC-MS) using a QExactive mass spectrometer coupled to an UltiMate 3000 RSLCnano System (Thermo Fisher Scientific). Briefly, $5 \mu \mathrm{L}$ of each sample was injected onto an Acclaim PepMap C18 $5 \mathrm{~mm} \times 0.3 \mathrm{~mm}$ i.d., $5 \mu \mathrm{m}$ particle size, $100 \AA$ pore size trapping column (Thermo Fisher) using $2 \%$ acetonitrile (LC-MS grade, Honeywell) (v/v) $+0.05 \%$ trifluoroacetic acid (VWR) as the solvent at a $120 \mu \mathrm{L} / \mathrm{min}$ flow rate for $0.25 \mathrm{~min}$. Subsequently, the valve was switched onto the analytical column. Peptides were separated using an Acclaim PepMap RSLC C18, $15 \mathrm{~cm}$ $\times 75 \mu \mathrm{m}$ i.d., $2 \mu \mathrm{m}$ particle size, $100 \AA$ pore size column (Thermo Fisher Scientific), and a linear gradient from 2.5 to $45 \%$ solvent B $(80 \%$ acetonitrile $\mathrm{v} / \mathrm{v}+0.1 \%$ formic acid $\mathrm{v} / \mathrm{v})$ over $10.75 \mathrm{~min}$. Solvent A was $0.1 \%$ formic acid in water (Honeywell) (v/v). A washing step of 3 min $99 \%$ solvent B was included and afterward, the column was re-equilibrated for $6 \mathrm{~min}$ at $2.5 \%$ solvent $\mathrm{B}$. The complete gradient was operated at $0.6 \mu \mathrm{L} / \mathrm{min}$. The total run time was $20 \mathrm{~min}$. The column oven temperature was set to $50{ }^{\circ} \mathrm{C}$. The LC system was connected to the mass spectrometer via a nanospray ion source. The mass spectrometer was operated in positive data-dependent acquisition mode and MS full scans were acquired at a resolution of 70.000 and a $200-2000 \mathrm{~m} / z$ range. MS full scans were followed by MS2 acquisition of the five most intense ion signals at a resolution of 17.500 and a scan range of $200-2000 \mathrm{~m} / z$. Ions were fragmented using higher collision-induced dissociation (HCD) and a normalized collision energy (NCE) of 25. The automatic gain control (AGC) target and maximum injection time for MS scans were $5 \times 10^{6}$ and $250 \mathrm{~ms}$, and for MS2 $5 \times 10^{5}$ and $80 \mathrm{~ms}$, respectively. The isolation window was set to $2.0 \mathrm{Da}$ and dynamic exclusion for identified peptides was set to $2.0 \mathrm{~s}$.

2.6.1. Mass Spectrometry Data Processing. Mass spectrometry raw data files were processed using MaxQuant (version 1.6.17.0). ${ }^{37}$ Data files were searched against the human proteome (Uniprot Knowledgebase, 73.118 sequences). Variable modifications were set to oxidation ( $\mathrm{M}$ ) and acetylation ( $\mathrm{N}$-terminus). No fixed modifications were allowed. Label-free quantification was enabled as well as intensity-based absolute quantification (iBAQ) calculation. All other parameters were set to default. The "proteinGroups.txt" output table from MaxQuant was used for further data processing in Perseus software (version 1.6.14.0). ${ }^{38}$ Proteins, identified "only by-site" or by matching to the reverse decoy database were removed from the analysis. Furthermore, only proteins identified at a minimum of three biological replicates of one sample were retained for downstream analysis. Since $\mathrm{iBAQ}$ values from MaxQuant correlate with protein abundance within a sample, ${ }^{39}$ this metric was used for the detection of differential protein abundance between different PEM coatings. iBAQ values were $\log 2$-transformed and the missing values were imputed by inserting the value of " 10 ". Proteins were annotated using the Gene Ontology as well as the Kyoto Encyclopedia of Genes and Genomes (KEGG) pathway database.

2.7. Intracellular Protein Analysis (DigiWest). DigiWest was performed as recently described. ${ }^{40}$ Briefly, gel electrophoresis and blotting onto poly(vinylidene difluoride) (PVDF) membranes were performed using the NuPAGE system as recommended by the manufacturer (Life Technologies, Carlsbad, California). Blots were washed in PBST, proteins were biotinylated on the membranes using NHS-PEG12-Biotin $(50 \mu \mathrm{M})$ in PBST for $1 \mathrm{~h}$ followed by washing in PBST and drying. Individual sample lanes were cut into 96 molecular weight fractions $(0.5 \mathrm{~mm}$ each $)$ and proteins were eluted in 96-well plates using $10 \mu \mathrm{L}$ of elution buffer per well ( $8 \mathrm{M}$ urea, $1 \%$ Triton$\mathrm{X} 100$ in $100 \mathrm{mM}$ Tris $-\mathrm{HCl} \mathrm{pH}$ 9.5). Eluted proteins from each molecular weight fraction were loaded onto color-coded, neutravidincoated Luminex bead sets (MagPlex, Luminex, Austin, TX). Luminex bead sets (384) were employed and the protein-loaded beads from 4 different sample lanes were pooled into 1 bead-mix resulting in 3 bead-mixes for the 12 samples. The bead-mixes were sufficient for 63 antibody incubations (see Table S4). Aliquots of the DigiWest beadmixes (1/67th per well) were added to 96-well plates containing 50 $\mu \mathrm{L}$ per well assay buffer (blocking reagent for enzyme-linked immunosorbent assay (ELISA) supplemented with $0.2 \%$ milk powder, $0.05 \%$ Tween- 20 , and $0.02 \%$ sodium azide, Roche). Beads were briefly incubated in assay buffer and the buffer was discarded. Antibodies were diluted in assay buffer and $30 \mu \mathrm{L}$ were added per well. After overnight incubation at $15{ }^{\circ} \mathrm{C}$ on a shaker, bead-mixes were washed twice with PBST and phycoerythrin (PE)-labeled secondary antibodies (Dianova) were added and incubated for $1 \mathrm{~h}$ at $23^{\circ} \mathrm{C}$. The beads were washed twice prior to the readout on a Luminex FlexMAP 3D. Secondary antibodies were either diluted in assay buffer or a polymer buffer (blocking reagent for ELISA supplemented with $4 \%$ poly(vinylpyrrolidone) (PVP) 360.000, 1\% milk powder, 0.05\% Tween20 , and $0.02 \%$ sodium azide, Roche). For quantification of the antibody-specific signals, the DigiWest analysis tool (version 3.8.6.1, Excel-based) was employed. This tool uses the 96 values for each initial lane obtained from the Luminex measurements on the 96 molecular weight fractions, identifies the peaks at the appropriate molecular weight, calculates a baseline using the local background, and integrates the peaks. The values are based on relative fluorescence (accumulated fluorescence intensity, AFI). For analysis, data (measured signal intensity) was median centered, normalized to the median measured signal intensity corresponding to the sample, median centered, and log 2-transformed. The software package MEV 4.8.1 was used for data visualization, clustering, and nonparametric statistical analysis. ${ }^{41}$

2.8. Statistical Analysis. Apart from the intracellular protein analysis using DigiWest, all statistical analysis and plotting were performed with GraphPad Prism 9.0 (GraphPad Software, San Diego). Statistical tests were applied as indicated in the respective figure legends, with a significance threshold of $p \leq 0.05$. For all tests comparing three or more groups, the recommended correction for multiple comparisons was applied. All graphs show mean \pm SD. 
A

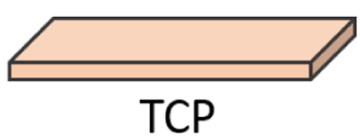

B Thickness

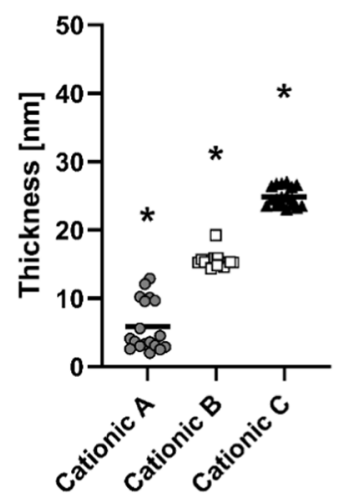

C

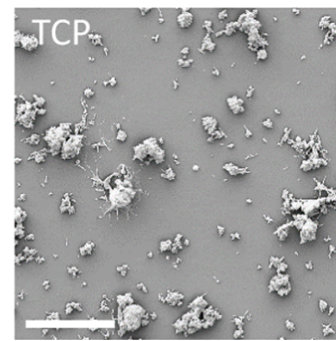

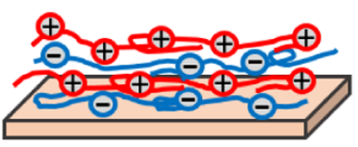

Cationic A

Surface charge
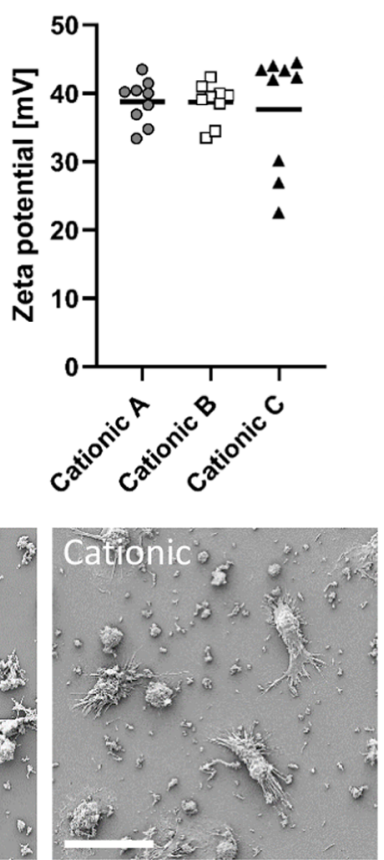

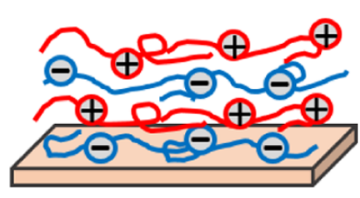

Cationic B

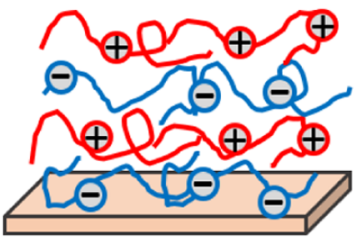

Cationic C

Protein adsorption
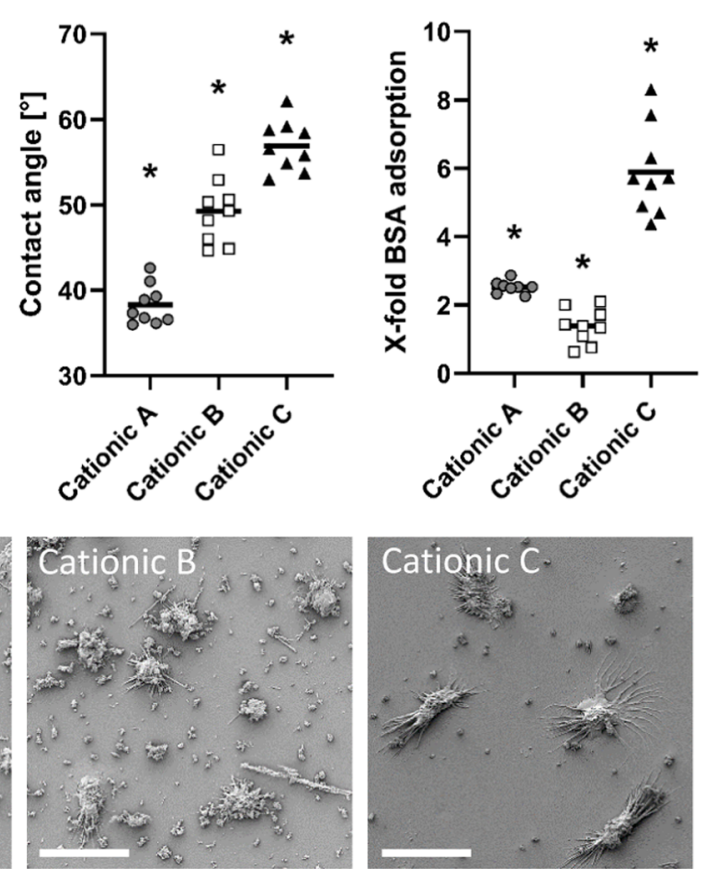

Figure 1. Physicochemical characteristics of the PEM coatings. Three different PEM coatings cationic A-C were generated by layer-by-layer deposition of polystyrene sulfonate (PSS) and polyallylamine hydrochloride (PAH) polyelectrolytes by applying different $\mathrm{pH}$ conditions during the assembly process. (A) Schematic overview of the deposited coatings. (B) Surface thickness (spectroscopic ellipsometry), surface charge (Zetasizer), and wettability (water contact angle) were assessed. Adsorption of BSA was quantified by measuring the fluorescence of adsorbed FITC-labeled BSA. Intensities of BSA adsorption were normalized to the FITC signal of polystyrene-adsorbed BSA, which was set to a value of 1 . $n=3$ experiments and $n=18$ total measurements for ellipsometry and $n=3$ experiments and $n=9$ total measurements for all other experiments. Lines represent the mean value. The ordinary one-way analysis of variance (ANOVA) was applied to compare all PEMs and $*$ indicates $p<0.05$ vs all other surfaces. (C) Scanning electron microscopy images of the three PEM coatings and uncoated TCP after incubation with PBMCs for 24 h. Scale bar $=20 \mu \mathrm{m}$.

\section{RESULTS}

3.1. Physicochemical Characteristics of Material Surfaces Can Be Modulated with Polyelectrolyte Multilayers. To investigate the effect of surface properties on protein adsorption and immune response, PEM coatings that were based on identical polyelectrolyte substrates but differed in selected physicochemical properties were applied on tissue culture plate (TCP) surfaces. By adjusting the $\mathrm{pH}$-sensitive charge density of the assembling polymers, three variations of PEM coatings were created, all based on alternating layers of the polyanion polystyrene sulfonate (PSS) and the polycation polyallylamine hydrochloride $(\mathrm{PAH})$. A schematic view of the applied coatings and their properties can be seen in Figure 1A. In reference to the polycationic coating termination, they are referred to as PEM coating cationic A, B, and C. Although the number of applied polyelectrolyte layers was identical for all three coatings, the increasing $\mathrm{pH}$ of the assembly solution from coatings $\mathrm{A}$ to $\mathrm{C}$ resulted in progressively increased thickness, ranging from $5.9 \pm 3.7 \mathrm{~nm}$ for coating cationic A to $24.9 \pm 1.4$ $\mathrm{nm}$ for cationic $\mathrm{C}$ as the thickest coating (Figure 1B). As parameters like surface charge and wettability can affect protein adherence, immune cell differentiation, and the immunological reactivity of a surface in general, these properties were determined employing water contact angle and Zetasizer analysis prior to assessing immunological effects. The mean surface charge was found to be identical for all coatings (Figure 1B). While all surfaces displayed hydrophilic properties, the cationic A coating was the most hydrophilic relative to the other two (contact angle $38.3 \pm 2.3^{\circ}$ ) (Figure 1B). This was followed by cationic $\mathrm{B}\left(49.3 \pm 3.9^{\circ}\right)$, while the least hydrophilic coating was cationic $\mathrm{C}\left(56.9 \pm 2.9^{\circ}\right)$. Analysis of protein adsorption on these three PEM coatings showed clear differences in the amount of BSA that was adsorbed onto the surface. The most BSA was found on cationic $\mathrm{C}$ with a 5.9 \pm 1.3 -fold increase over the uncoated polystyrene (PS) control. In comparison, PEMs cationic A and B showed a $2.5 \pm 0.2$ and $1.4 \pm 0.5$-fold increase, respectively, compared to the uncoated control (Figure 1B). 
A

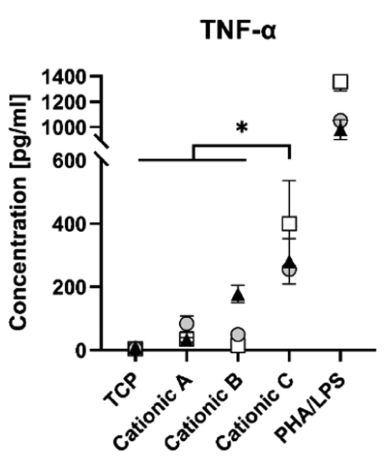

MCP-1

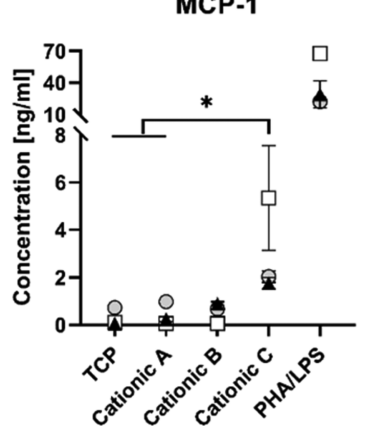

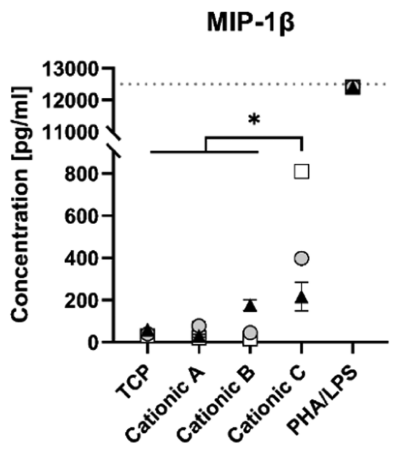

IL-6

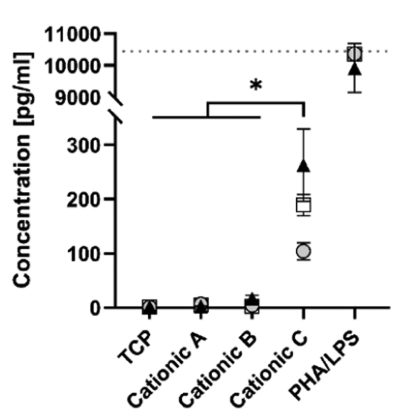

B

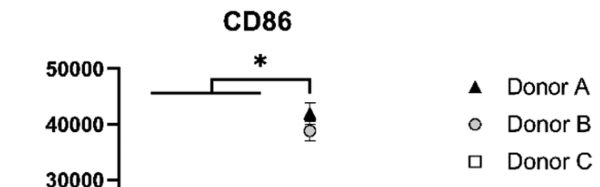

业
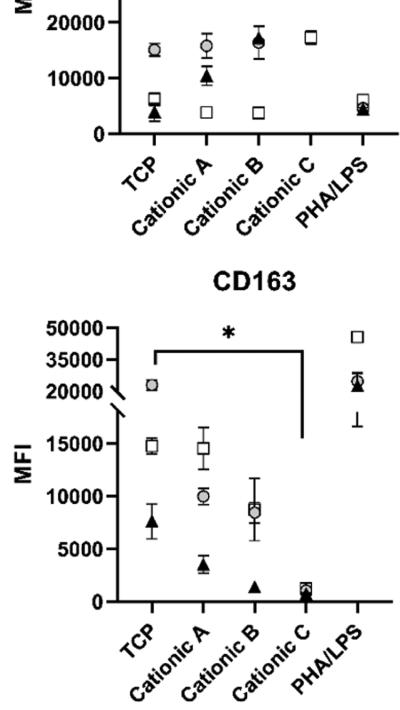

Figure 2. Proinflammatory cytokine secretion and expression of surface markers on PBMCs cultured on PEM surfaces. PBMCs from three donors were cultured for $72 \mathrm{~h}$ on PEM surfaces cationic A, B, and C. TCP served as a negative control and stimulation with LPS and PHA as a positive control. (A) Concentration of proinflammatory cytokines quantified in the cell culture supernatant using multiplex assays. The dotted line indicates the upper limit of quantification. (B) Expression of cell surface markers CD86 (proinflammatory) and CD163 (anti-inflammatory) on monocytes analyzed by flow cytometry. Three donors were tested with two technical replicates for all conditions. Symbols represent mean \pm SD. RM one-way ANOVA compares the mean of each surface to the mean of every other surface (with the exception of the PHA/LPS condition). * indicates $p<$ 0.05. MFI: mean fluorescence intensity.

To gain an initial insight into the interaction of immune cells with these coated surfaces, we cultured PBMC on the surfaces cationic $\mathrm{A}, \mathrm{B}$, and $\mathrm{C}$ for $24 \mathrm{~h}$ and visually inspected their interaction with the coatings using scanning electron microscopy. The images revealed uniform surfaces allowing cell adhesion with different morphologies but apparently a tight interaction to the smooth PEMs (Figure 1C). No consistent differences in cell morphologies between the different surfaces investigated could be observed. The structure of the deposited PEMs was quite homogeneous and no irregularities could be observed in the micrometer range. Ellipsometric measurements at 18 positions of three independent samples showed minor variations in the coating thickness for cationic B and C (coefficient of variation 7\% and $6 \%$, respectively). Only the thinnest coating cationic A showed higher variations between 2 and $13 \mathrm{~nm}$ (Figure 1B). Taken together, the three coatings differ in their nanometer thickness, wettability, and ability to adsorb proteins, while having a comparable microstructure and surface charge.

3.2. More Hydrophobic PEM Coating Cationic C Elicits the Highest Proinflammatory Immune Response. Having observed that immune cells made contact with and interacted with the PEM surfaces using electron microscopy, we next investigated the biological effects in response to these surfaces. To do this, PBMCs were incubated for $72 \mathrm{~h}$ on the three different coatings prior to the assessment of cytokine production in the cell culture supernatant and $\mathrm{CD}$ molecule expression on cellular immune populations. We observed the more hydrophobic cationic $\mathrm{C}$ surface to result in an elevated secretion of proinflammatory cytokines TNF- $\alpha$, MIP- $1 \beta$, and IL-6 compared to all other surfaces (Figure 2A). Similar results were also found for MCP-1, with a significantly higher level produced in response to cationic $\mathrm{C}$ compared to cationic $\mathrm{A}$ and the TCP control. Cytokine levels for cationic A and B did not differ from those found in response to the uncoated TCP control. Similar trends were observed for additionally investigated proinflammatory cytokines IL- $1 \beta$, IFN- $\gamma$, and IL8 (Figure S3). All anti-inflammatory cytokines examined either showed no change between PEM surfaces (IL-10, IL-1RA, VEGF) or were below the limit of quantification (IL-4, IL-13; data not shown). When investigating the expression of surface markers by flow cytometry, we found the observed cytokine response to be accompanied by the alteration of $\mathrm{CD}$ molecules on monocytes. Expression of the proinflammatory marker CD86 was substantially increased in response to PEM cationic $\mathrm{C}$ compared to coatings $\mathrm{A}$ and $\mathrm{B}$ and the TCP control (Figure $2 B)$. In line with this, expression of the anti-inflammatory marker CD163 was the lowest on cationic C and was the only condition significantly lower than the negative control TCP. Levels of CD86 and CD163 in response to the positive control LPS/PHA were altered in accordance with the literature. ${ }^{42}$ Other cell populations investigated, $\mathrm{T}$ and NK cells, did not show any noteworthy changes in the surface marker expression across the three PEM surfaces (data not shown). In summary, we observed the strongest immune response in contact with the more hydrophobic PEM cationic C, while the effects of coatings $\mathrm{A}$ and $\mathrm{B}$ were mild to moderate in comparison. 


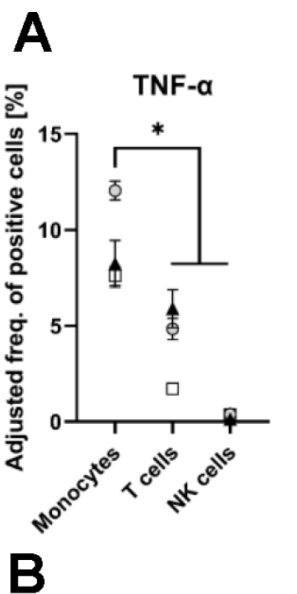

TNF- $\alpha^{+}$Monocytes
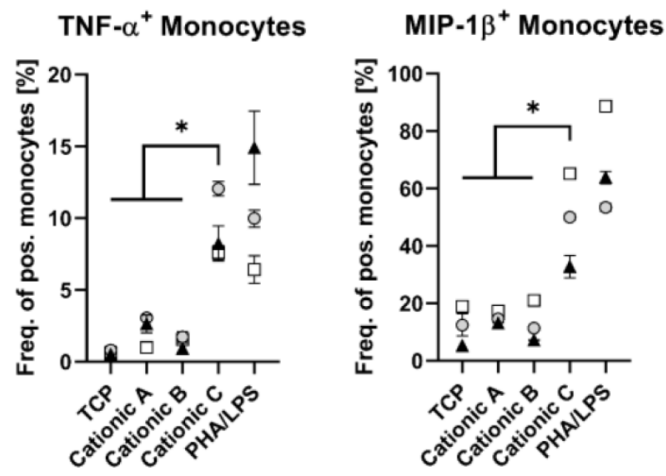

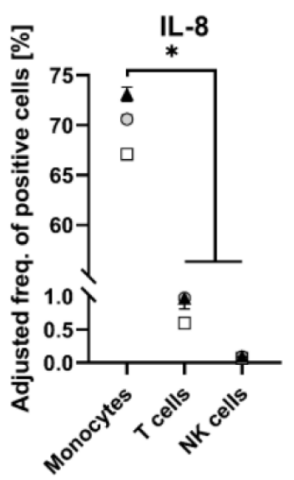

IL- ${ }^{+}$Monocytes
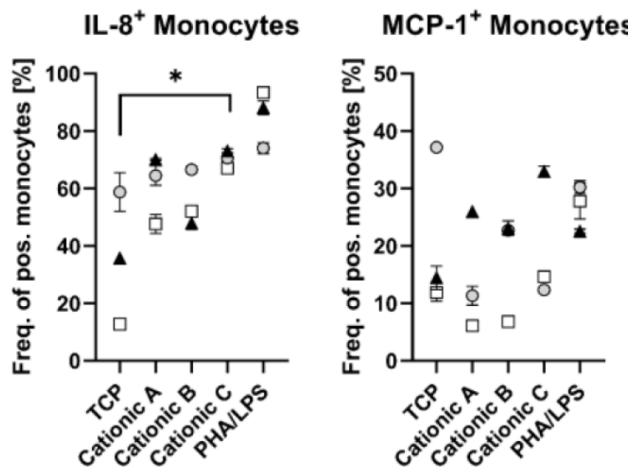

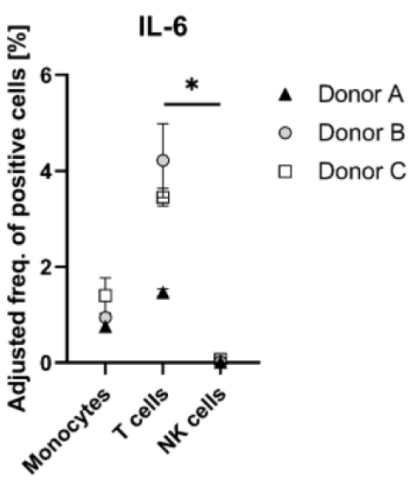

IL- $6^{+}$Monocytes

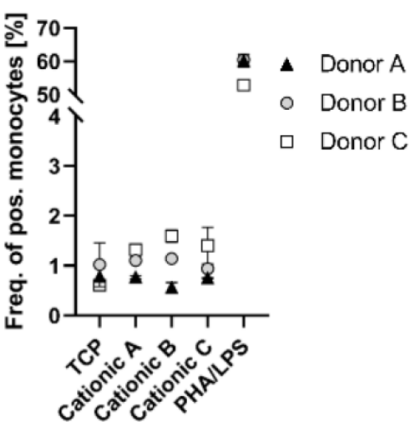

C

TNF- $\alpha$ Monocytes

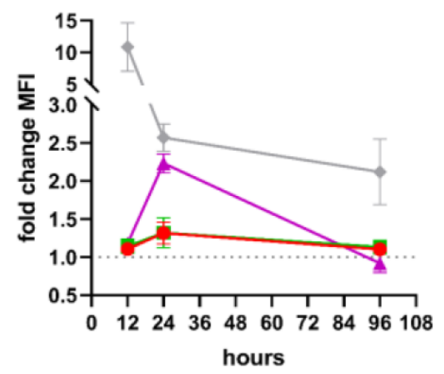

MIP-1 $\beta$ Monocytes

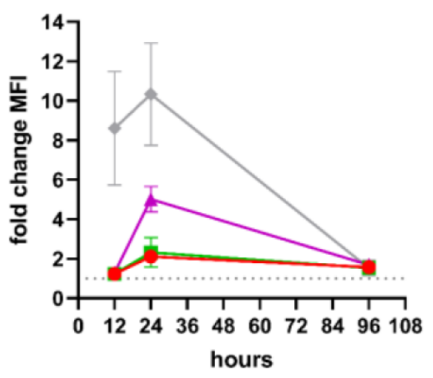

IL-8 Monocytes

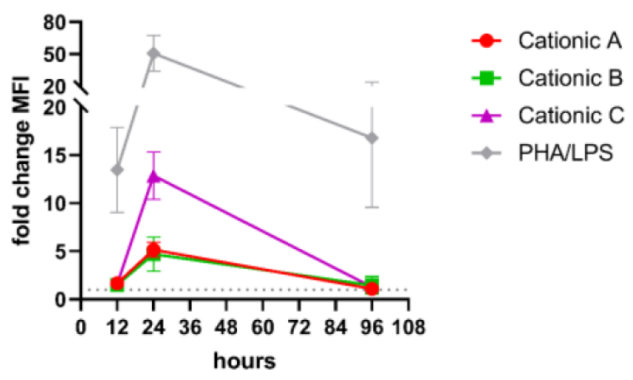

Figure 3. Intracellular cytokine analysis after cultivation of PBMCs on PEM coatings. PBMCs were cultured on PEM coatings cationic A to C and the uncoated TCP control. Intracellular staining was performed for the proinflammatory cytokines TNF- $\alpha$, MIP-1 $\beta$, IL-8, MCP-1, and IL-6 followed by flow cytometric analysis. (A) Frequency of positive cells for monocytes, $\mathrm{T}$ cells, and NK cells after cultivation of PBMC on PEM cationic $\mathrm{C}$ for $24 \mathrm{~h}$. Frequencies of positive $\mathrm{T}$ cells and NK cells were adjusted to account for the differences in the relative size of these populations in relation to monocytes. $n=3$ donors and two technical replicates. RM one-way ANOVA was applied to compare the mean of every population to the mean of every other population. * indicates $p<0.05$. (B) Frequency of positive monocytes of three donors tested in duplicates. RM one-way ANOVA compares the mean of coating cationic $\mathrm{C}$ to the mean of surfaces cationic $\mathrm{A}$, cationic $\mathrm{B}$, and the TCP control. * indicates $p<0.05$. (C) MFI of monocytes was quantified after culturing PBMCs for 12, 24, and $96 \mathrm{~h}$. PBMCs of one donor in duplicate tested in two separate experiments. Graphs show fold change MFI of the TCP control (indicated by dotted lines) for cationic A (circles, red), cationic B (squares, green), cationic C (triangles, purple), and the positive control PHA/LPS (diamond, gray). All graphs show mean \pm SD.

3.3. Proinflammatory Immune Response of PEM Cationic C Is Mediated through Monocytes and Peaks after $24 \mathrm{~h}$. The results obtained by examining cytokine secretion and $\mathrm{CD}$ molecule expression hinted that monocytes played a major role in the immune response to the PEM surfaces. To investigate this more closely, we next examined intracellular cytokine expression in several immune cell populations in PBMC. Having observed PEM cationic $\mathrm{C}$ to elicit the highest proinflammatory response, this surface was chosen for analysis. Investigating the expression of proinflammatory cytokines in monocytes, $\mathrm{T}$ cells, and NK cells showed very low frequencies of cells expressing TNF- $\alpha$, MIP-
$1 \beta$, IL- 8 and MCP- 1 in both NK cells and T cells but a significantly higher expression of these cytokines in monocytes (Figure 3A). Differences were most evident for IL-8 and MCP1, for which on average $70.3 \%$ and $20.0 \%$ of monocytes were positive, respectively, while less than $1 \%$ of $\mathrm{T}$ cells and $\mathrm{NK}$ cells were positive for these cytokines. Similar to previous findings from our group, ${ }^{32}$ IL- 6 was an exception to this trend and showed no difference in the frequency of positive cells between monocytes and $\mathrm{T}$ cells. This observation might be due to the low frequency of IL- 6 expressing cells for both of these populations or may more broadly reflect the ability of both monocytes and $\mathrm{T}$ cells to produce this molecule. ${ }^{2,43}$ Having 

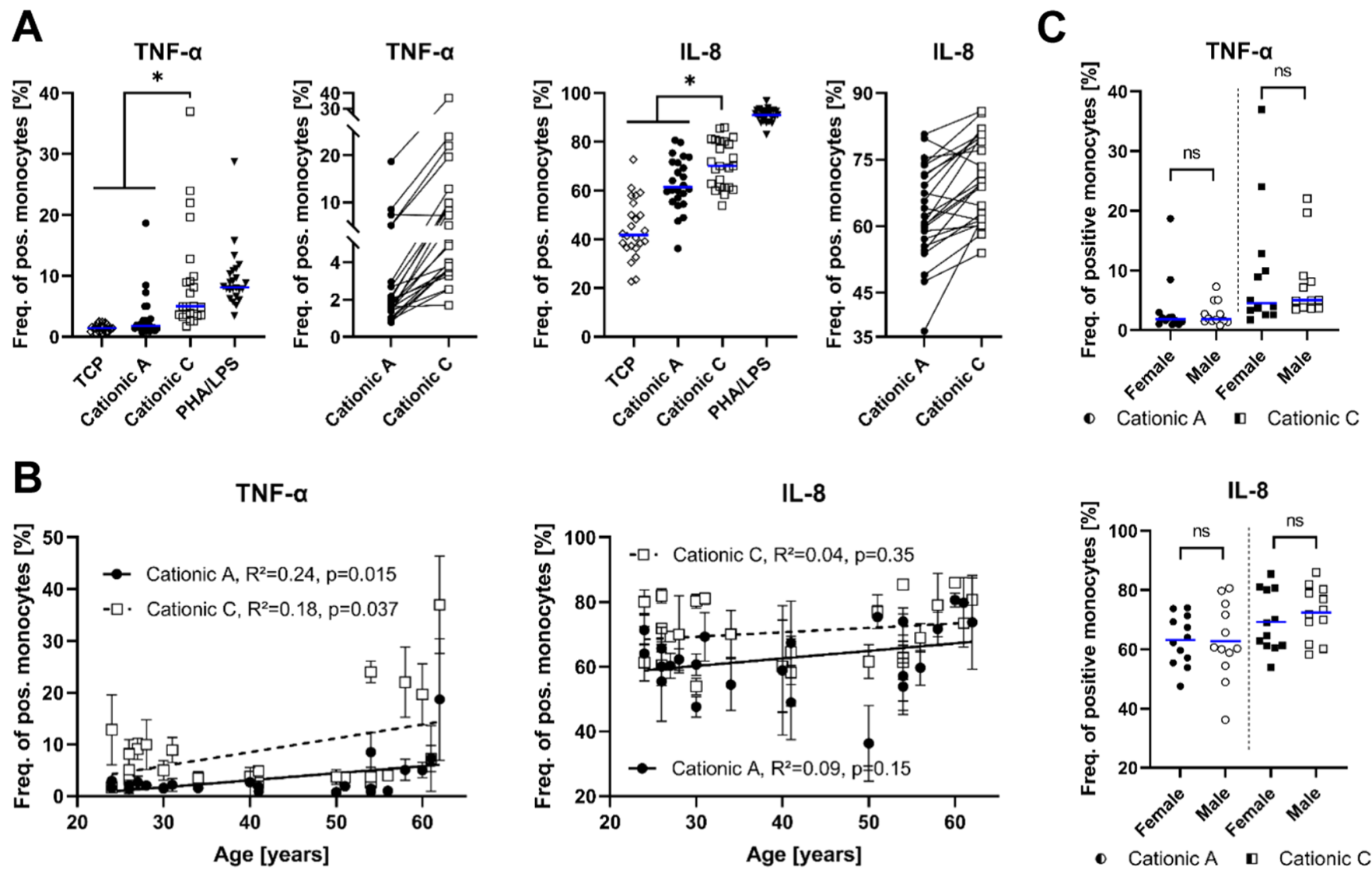

Figure 4. Intracellular expression of proinflammatory cytokines in monocytes in a cohort of 24 individuals. The frequency of TNF- $\alpha$ and IL-8 positive monocytes after $24 \mathrm{~h}$ of culture was quantified in three independent experiments using flow cytometry in 24 donors. (A) Comparison of cytokine expression on PEM cationic A and cationic C as well as TCP (negative control) and stimulated cells using PHA/LPS (positive control). RM one-way ANOVA was applied comparing cationic C vs cationic A and the TCP control, ${ }^{*} p \leq 0.05$. Small graphs: lines connect results of identical donors. (B) Age-dependent frequency of cytokine positive monocytes. Simple linear regression in a continuous line (cationic A) and dashed line (cationic C). (C) Analysis of PEMs cationic A and $\mathrm{C}$ with regard to sex. Female and male donors were compared for each surface separately using the unpaired $t$-test. Graphs in panels (A) and (C) show mean values and the blue line indicates the median. Panel (B) shows mean \pm SD.

identified monocytes as the main immune cell population driving the cytokine response, differences between the immune response to cationic $\mathrm{C}$ and the other surfaces were assessed in monocytes. Frequencies of TNF- $\alpha$ and MIP- $1 \beta$ positive monocytes were found to be strongly enhanced on PEM cationic $\mathrm{C}$ compared to all other PEM surfaces and the TCP control (Figure 3B). For IL-8, a similar trend was observed with a significant increase in IL-8 positive monocytes on cationic C compared to TCP. No clear trend was observable for MCP-1 and IL-6.

Cytokine release is a dynamic process that is subject to rapid regulatory mechanisms. To reveal the process of cytokine response toward the PEM coatings across time, cytokine production by monocytes was investigated after 12, 24, and 96 $\mathrm{h}$ of biomaterial contact. While PHA/LPS stimulated cells showed a strong increase for MIP-1 $\beta$, TNF- $\alpha$, and IL-8 expression already after $12 \mathrm{~h}$, no induction on the three PEM coatings was found at this time point (Figure 3C). In contrast, after $24 \mathrm{~h}$ of cultivation, cellular activation was observed on all PEM substrates. At this stage, coating cationic $\mathrm{C}$ showed the strongest cytokine expression resulting in a 2.2 (TNF- $\alpha$ ), 5.0 (MIP-1 $\beta$ ), and 12.9 (IL-8)-fold signal increase compared to the negative control, while this increased only $1.3 / 1.3$ (TNF$\alpha$ ), 2.1/2.3 (MIP-1 $\beta$ ), and 5.2/4.7 (IL-8)-fold for cationic A/ $\mathrm{B}$, respectively. After cultivation for $96 \mathrm{~h}$, the cytokine response was already declined and no differences in the cytokine expression across the PEM surfaces were observed. Cytokines MCP-1 and IL-6, which have already been shown in Figure 3B to not differ in their expression levels after $24 \mathrm{~h}$, were also unaffected by the different coatings after 12 and $96 \mathrm{~h}$ (Figure S4). Taken together, these results showed that mainly monocytes were responsible for cytokine release in response to the PEM coatings investigated and for this response to peak after $24 \mathrm{~h}$ of biomaterial contact. The results also confirm the cationic surface $\mathrm{C}$ to produce the highest proinflammatory response compared to the PEMs cationic $\mathrm{A}$ and $\mathrm{B}$.

3.4. Testing a Large Cohort Confirms the Results Obtained with a Smaller Number of Donors. Having characterized the immune responses to these PEM coatings in a small set of donors, we then cast a wider net and investigated a broader spectrum of biological variation by employing a large panel of donors. In particular, we were interested to know if the results observed for three randomly selected donors are representative also in a larger cohort and if donor-specific characteristics such as sex or age have any impact. To achieve this, we selected 24 healthy donors ( 12 female and 12 male) with ages ranging from 24 to 62 years (Table S1) and examined their response to the cationic $\mathrm{A}$ and $\mathrm{C}$ surfaces, as these represent the PEMs with the lowest (cationic A) and the highest (cationic C) degree of proinflammatory response 

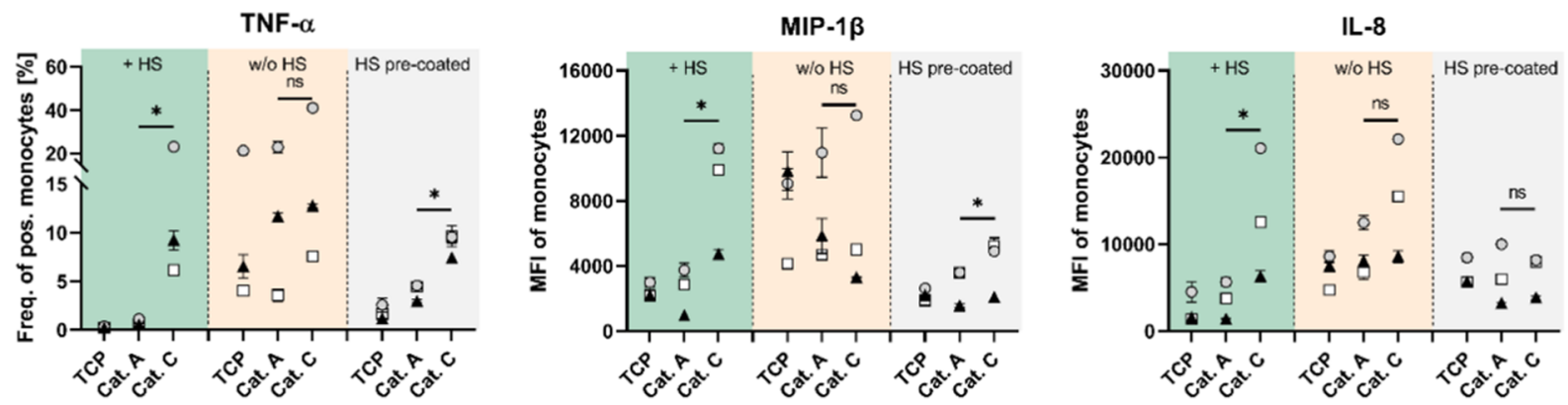

- Donor A

- Donor B

- Donor C

Figure 5. Influence of human serum on proinflammatory cytokine expression by PBMCs cultured on PEM coatings. PBMCs were cultured on the TCP control, PEM cationic A and PEM cationic C in human serum-containing media (+HS, green), serum-free media (w/o HS, orange), or in serum-free media after precoating of the surface with serum for $1 \mathrm{~h}$ (HS precoated, gray). The frequency of positive monocytes or MFI was quantified for TNF- $\alpha$, MIP-1 $\beta$, and IL-8 in three healthy donors with three technical replicates. Culturing period was 24 h. $t$-test was applied to compare surfaces cationic $\mathrm{A}$ and $\mathrm{C}$ within the respective culturing groups. ${ }^{*} p \leq 0.05$. All graphs show mean $\pm \mathrm{SD}$.
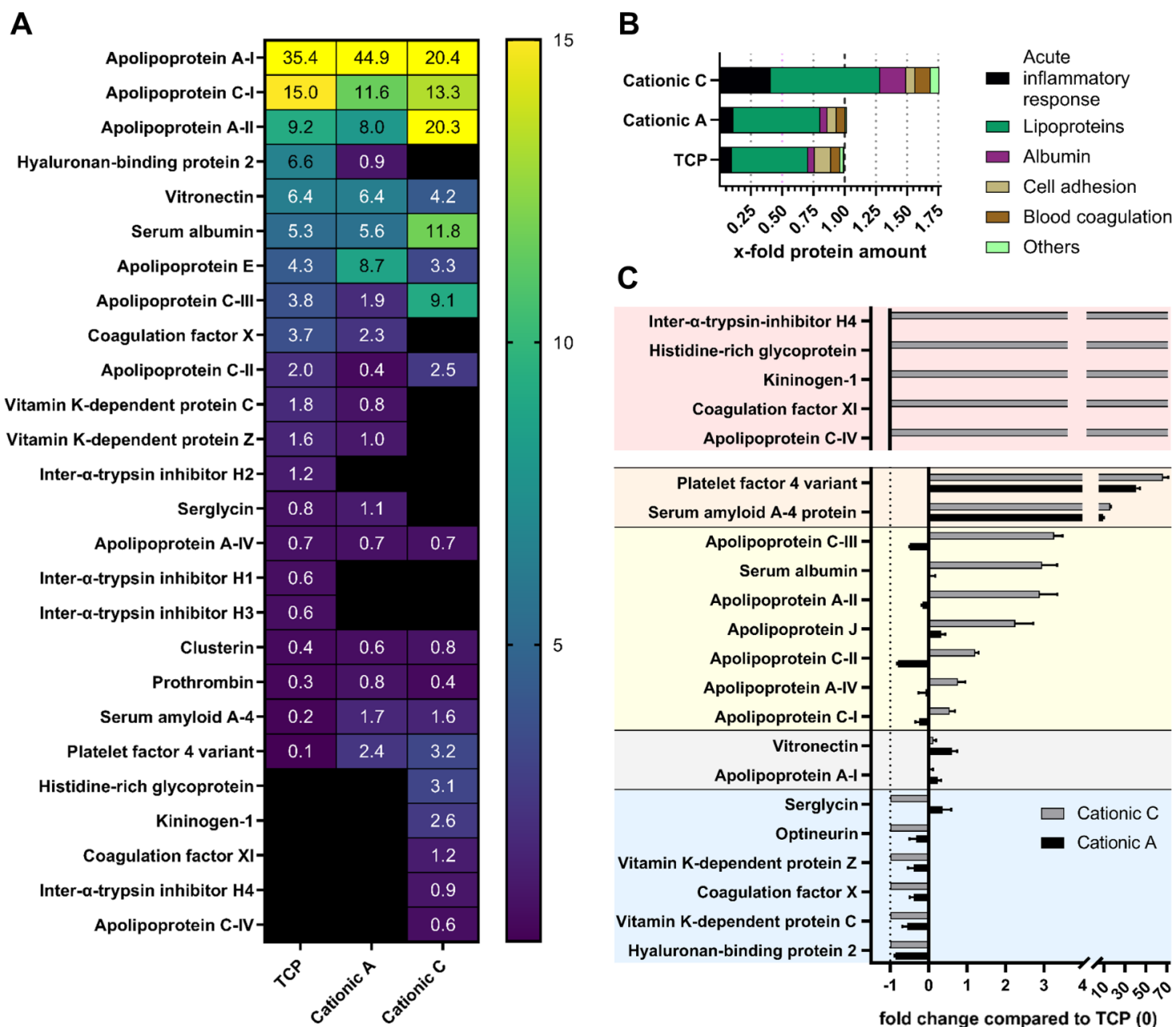

C
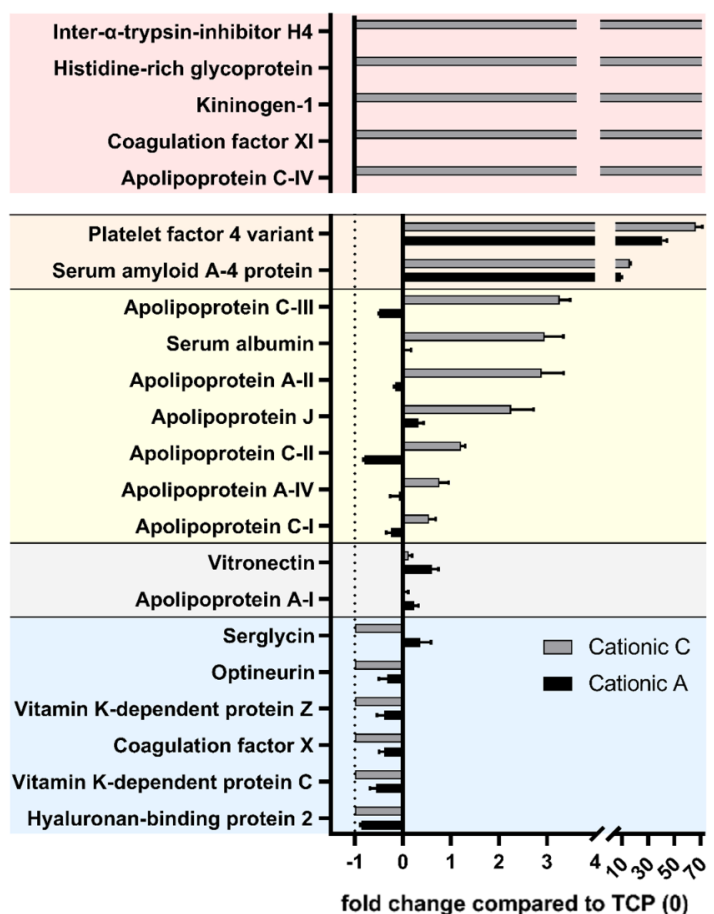

Figure 6. Quantification of protein adsorption. PEM surfaces cationic A, cationic C, and the TCP control were incubated for $1 \mathrm{~h}$ with media containing 10\% human serum. Adsorbed proteins were determined by LC-MS with $n=3$ for all surfaces. (A) Heatmap of all proteins identified with an abundance $\geq 0.5 \%$ on one of the surfaces' minimum. Values indicate the abundance percentage (total protein abundance is $100 \%$ on each surface). (B) Classification of adsorbed proteins according to their function in an $x$-fold protein amount of TCP (TCP normalized to 1). PEM cationic C 1.76-fold protein amount of TCP and PEM cationic A 1.02-fold protein amount of TCP. (C) Proteins with significantly different protein amounts on the two PEM coatings (orange, yellow, and gray backgrounds) or being present on cationic C only (red) or cationic A only (blue background) are depicted as fold change expression of TCP (TCP set to 0 , level indicated by a continuous black line). Reduction by -1 (dashed line) indicates that the respective protein was not found on the corresponding surface. Multiple $t$-tests were applied $(p \leq 0.05)$. Graph shows mean \pm SD.

observed. We observed levels of TNF- $\alpha$ and IL-8 by this cohort of 24 individuals to be broadly representative of the results obtained with the initial three donors. Both cytokines were found at higher levels in response to the cationic $\mathrm{C}$ surface compared with cationic A and the TCP control $(p<$ 0.05 for both cytokines) (Figure 4A). However, a small 
number of outliers did not fit the trend seen for the rest of the cohort. Three donors (representing $12.5 \%$ of the total cohort) were observed to show a slightly lower frequency of IL-8 positive monocytes for the cationic $\mathrm{C}$ surface compared to cationic A (see Figure 4A far right graph). For one of these donors, this was also true for TNF- $\alpha$ while the other two donors showed the same trend as the rest of the cohort for this marker. The outliers were not related in sex or age (two females and one male; 24, 41, and 61 years old) and were not included in other experiments. Next, we investigated whether there was an association between the features of the donors and the observed immune responses. A weak age-dependent increase in the number of TNF- $\alpha$ positive monocytes for both PEM surfaces (cationic A: $R^{2}=0.24, p=0.015$; cationic C: $R^{2}$ $=0.18, p=0.037$ ) was observed (Figure 4B). No agedependent correlation was found for IL-8 $\left(R^{2}=0.09, p=0.15\right.$; $\left.R^{2}=0.03, p=0.35\right)$ or in the negative and positive control groups (Figures $4 \mathrm{~B}$ and S5). Separating the donors by sex showed no differences between the median values of male and female donors for any cytokine or surface tested (Figure 4C). Taken together, these findings confirm our previous results also for a larger cohort while indicating occasional donorspecific differences.

3.5. Presence of Serum Influences the Immune Response to PEM Coatings. Having performed a suite of comprehensive analyses characterizing the immune response and identifying PEM cationic $\mathrm{C}$ as the most inflammatory coating, we next aimed to investigate potential causes of the differences in proinflammatory responses observed for the different PEM coatings tested. It is known that the immune response can be modulated by the adsorbed layer of proteins on a surface, which is in turn influenced by the surface properties of that material. ${ }^{10,11,14}$ To analyze the influence of serum proteins on the observed immune response to our PEM coatings, we compared immune responses of PBMC cultured in standard cell culture media containing human serum with cultures of serum-free media. We observed the immune response to be dependent on the presence of proteins. In human serum-containing cultures (+HS, green), a significantly higher cytokine release on cationic $\mathrm{C}$ was observed for all three cytokines TNF- $\alpha$, MIP- $1 \beta$, and IL- 8 compared to cationic A (Figure 5), as previously observed. However, when the same PEM surfaces were cultured without serum, no differences in the degree of cytokine release were found (w/o HS, orange). Interestingly, precoating the surfaces with human serum prior to culture with serum-free media (HS precoated, gray) produced similar immune responses to those observed in the presence of serum-containing media, with elevated levels of TNF- $\alpha$ and MIP- $1 \beta$ in response to cationic C compared to the cationic A surface. It is worth noting that in the absence of serum, proinflammatory responses to TCP and cationic A surfaces were found to be generally elevated compared to serum-containing cultures. Together, these results suggest a role for absorbed serum proteins in modulating the immune response to material surfaces of different wettabilities.

3.6. Adsorption of Acute Inflammatory Response Proteins Is Enhanced on More Hydrophobic PEM Cationic C. To investigate if the adsorbed protein layer differs on the two PEM surfaces and if these differences could be a cause for the distinct immune response observed between the coatings, the composition of the protein layers was analyzed following contact with human serum. PEMs cationic $\mathrm{A}$ and $\mathrm{C}$ were incubated with media containing $10 \%$ human serum, and surface proteins were quantified after on-surface trypsin digest using LC-MS/MS. In total, 26 proteins were identified on the two PEM surfaces (criteria: abundance $\geq 0.5 \%$ ), with changes in the type and quantity of proteins detected when comparing cationic A with cationic C (Figure $6 \mathrm{~A})$. Among the most abundant proteins were several lipoproteins that were found on all three surfaces, with surface-dependent differences for the specific type of lipoprotein. While adsorption of albumin had an abundance of $5.3 \%$ and $5.6 \%$ for the TCP control and PEM cationic A, respectively, a higher abundance of albumin was observed for PEM cationic C with $11.8 \%$. The total amount of serum proteins adsorbed was clearly enhanced on PEM cationic C (1.76-fold protein amount compared with TCP), while the total amount on PEM cationic A was similar to that in the TCP control (1.02-fold protein amount of TCP) (Figure 6B). To analyze the characteristics of the adsorbed protein layers, identified proteins were grouped based on Gene Ontology database (GOBP) functions. Lipoproteins were the main class of adsorbed proteins on all surfaces followed by proteins involved in the inflammatory response (Figure 6B). Proteins involved in other roles such as cell adhesion and blood coagulation were found in small amounts only. The comparison between surfaces cationic $A$ and $C$ showed a 4fold increase in the amount of acute inflammatory response proteins on surface cationic $C$ ( 0.41 -fold protein amount on cationic $C$ vs 0.11 -fold protein amount on cationic A). This resembles the observed immune response.

To investigate potential differences between the two PEM coatings more closely, statistical analysis was applied to all proteins identified. The identified proteins could be subdivided into five groups (Figure 6C). The red group includes proteins that can only be found on surface cationic $\mathrm{C}$ but not cationic $\mathrm{A}$ (inter- $\alpha$-trypsin inhibitor heavy chain $\mathrm{H} 4$, histidine-rich glycoprotein, kininogen 1 , coagulation factor XI, apolipoprotein C-IV). The majority of proteins of this group are involved in regulatory roles like blood coagulation and angiogenesis. Proteins in the orange group are increased on both PEM coatings compared to the TCP surface with a statistically significant higher abundance on cationic $\mathrm{C}$ (platelet factor 4 variant and serum amyloid A4 (SAA4)). Notably, serum amyloids are classical acute-phase proteins that are known to induce the expression of proinflammatory cytokines TNF- $\alpha$, IL- $1 \beta$, and IL- 8 in neutrophils and monocytes. ${ }^{44,45}$ Proteins in the yellow protein group were found to be strongly increased on surface cationic $C$, while being at or below the level of the TCP control for cationic A, showing significant differences between the two PEM coatings cationic $\mathrm{C}$ and $\mathrm{A}$ (apolipoprotein C-III, serum albumin, apolipoprotein A-II, apolipoprotein J, apolipoprotein C-II, apolipoprotein A-IV, apolipoprotein C-I). Remarkably, the vast majority of proteins in this group were found to be apolipoproteins. The observation that these proteins are present in a greater amount on cationic $\mathrm{C}$ fits with the observation of this coating producing higher proinflammatory responses as some of these apolipoproteins have been associated with proinflammatory responses (see Section 4). ${ }^{46-50}$ The gray group in Figure $6 \mathrm{C}$ includes proteins with statistically elevated protein amounts on cationic A (vitronectin and apolipoprotein A-I). Apolipoprotein A-I is known for its anti-inflammatory effects on monocytes where it inhibits the production of proinflammatory cytokines TNF- $\alpha$ and IL- $1 \beta^{51}$ and is the most abundant protein on the PEM cationic A, accounting for $44.9 \%$ of the 


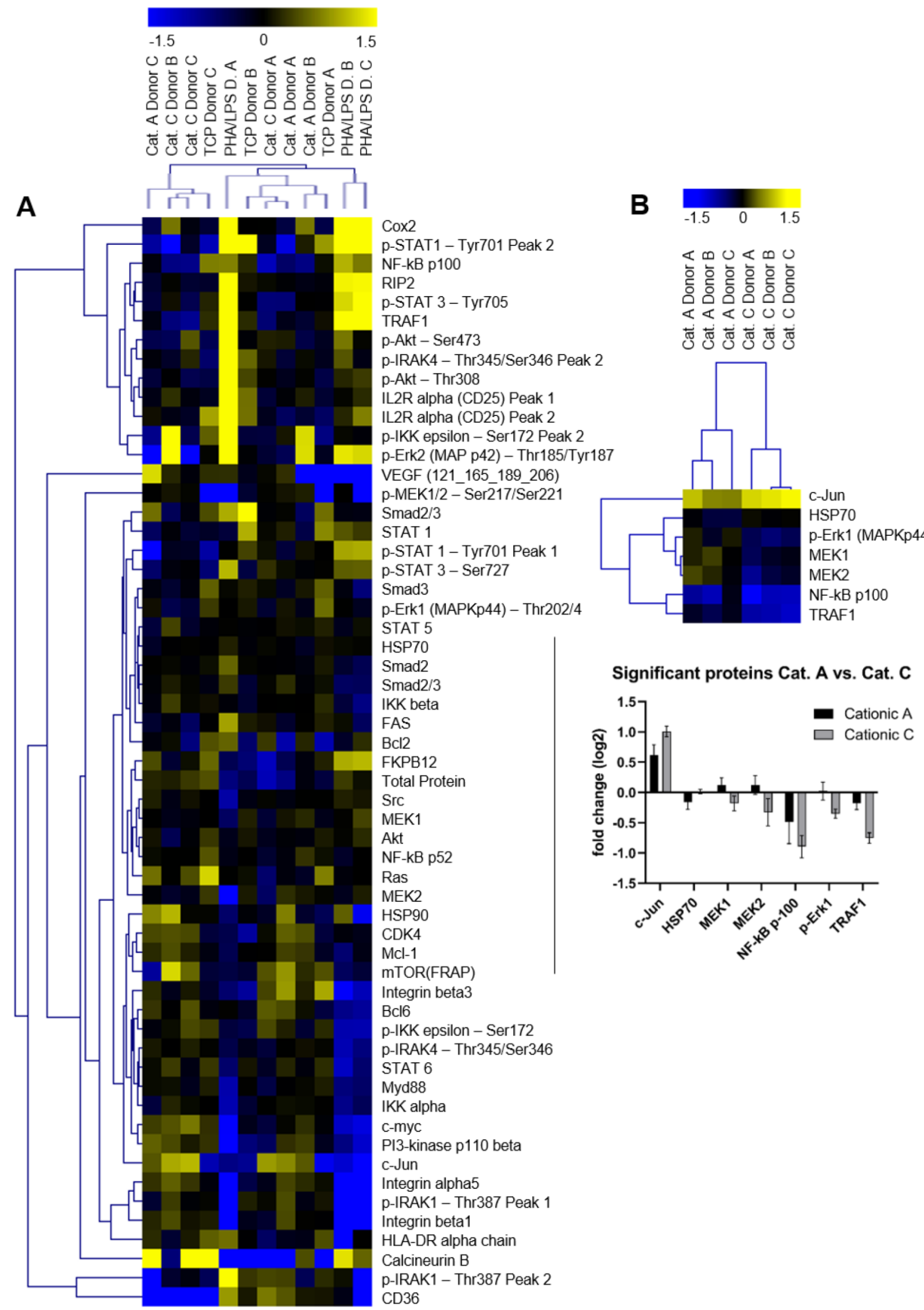

Figure 7. DigiWest-based protein expression analysis of intracellular signaling molecules in monocytes. Monocytes were isolated from the PBMC cell suspension using MACS isolation and cultured for $24 \mathrm{~h}$. Protein expression of 63 cell signaling proteins and their modifications were investigated in monocytes of $n=3$ different donors using DigiWest. (A) Hierarchically clustered protein expression of all data (Euclidean distance and complete linkage clustering). Color gradient from blue to yellow corresponds to low or high antibody-specific signals. (B) Proteins with a significant (nonparametric Wilcoxon rank-sum test, $p \leq 0.05$ ) difference in expression levels comparing cationic $\mathrm{A}$ and cationic $\mathrm{C}$ are shown as a hierarchically clustered heatmap (Euclidean distance and complete linkage clustering). Bar chart indicates the log fold change of significant proteins. Graph shows mean \pm SD. 
total protein amount (see Figure 6A). On the PEM cationic C, apolipoprotein A-I only accounts for $20.4 \%$ of the total protein amount. Thus, this finding is in line with the immunological results showing a lower cytokine response for cationic A compared to cationic C. The last group in Figure 6C (blue) shows proteins being present on cationic A but not cationic $\mathrm{C}$. As these proteins were all identified on TCP as well, this shows a high similarity between surfaces cationic A and TCP, which was mirrored in the type of immune response that they provoked. Numerical values for data in Figure 6 can be found in Tables S2 and S3.

Taken together, proteins found to a higher extent and in large relative abundance on cationic $\mathrm{C}$ are acute inflammatory response proteins (e.g., SAA4) or are associated with inflammatory responses based on the literature (e.g., apolipoprotein A-II and apolipoprotein C-III), while the most abundant protein on cationic $\mathrm{A}$ is related to antiinflammatory processes (apolipoprotein A-I). Thus, these observations may relate to differences in the degree of inflammatory responses found to these surfaces.

\subsection{Intracellular Levels of Mitogen-Activated Protein} Kinase (MAPK) Proteins in Monocytes Are Associated with the Immune Response to PEM Coatings. After having observed differences in the protein adsorption and the proinflammatory response to the PEM surfaces, we next investigated immune cells regarding intracellular signaling pathways. We were interested in identifying changes in these pathways, which could be linked to the observed immune responses. Previous experiments (Figure 3) had shown the immune response to be primarily driven by monocytes. To identify modulation of intracellular pathways in these cells, we cultured an isolated population of monocytes on the three surfaces cationic A, C, and the TCP control. After $24 \mathrm{~h}$ of culture, the expression of 63 intracellular signaling proteins was investigated using the DigiWest technology, which allows detection of both protein abundance as well as phosphorylation status. A heatmap of all analyzed proteins including their fold change in expression can be seen in Figure 7A (for raw data, see Table S4). Comparison between PEMs cationic $A$ and $C$ showed statistically significant differences in the expression of seven proteins (Figure 7B). Strikingly, six out of the seven signaling proteins identified are part of the MAPKsignaling pathways, indicating a general role of this pathway in response to PEM surfaces. Out of these, c-Jun was found to be elevated in monocytes in response to cationic $\mathrm{C}$, while proteins MEK1, MEK2, NF- $\kappa \mathrm{B}$, p-Erk1, and TRAF1 were expressed at lower levels in response to cationic $\mathrm{C}$ compared to cationic $\mathrm{A}$. It should be highlighted that qualitative differences in the response of these proteins were identified in addition to the previously mentioned differences in fold expression. As such, MEK1, MEK2, NF- $\kappa \mathrm{B}$, and Erk $1 / 2$ increased in response to cationic $\mathrm{A}$, while they were downregulated by cationic $\mathrm{C}$. The only non-MAPK protein differing between cationic $\mathrm{C}$ and $\mathrm{A}$ was the molecular chaperone HSP70, which showed a higher expression on cationic $\mathrm{C}$ compared to cationic A. Comparison of negative (TCP) and positive controls (PHA/LPS) revealed changes in the expression and phosphorylation of 20 of the analyzed proteins (Figure S6). As reported in the literature, pharmacological stimulation of monocytes with PHA/LPS (positive control) showed a strong increase in COX2 and activation of MAPK and JAK/STAT signaling pathways. ${ }^{52-54}$

\section{DISCUSSION}

In this study, the relationship between biomaterial surface properties, adsorbed proteins, and the resultant immune response was investigated. The use of custom-modified surfaces, human serum, and a human-based primary immune cell model enabled a comprehensive analysis of the interactions between these complex aspects. We observed a materialdependent secretion of proinflammatory cytokines in response to different PEM coatings, which was largely due to monocytes. Differences in the immune response were found to be only apparent in the presence of human serum. Furthermore, we observed stronger proinflammatory responses to be associated with the adsorption of apolipoproteins A-II and C-III on the surface of the more hydrophobic PEM. Conversely, the adsorption of apolipoprotein A-I was associated with the most hydrophilic PEM coating that provoked only a mild inflammatory response.

The targeted modification of surfaces is regarded as a possible way to specifically alter the immune response to biomaterials. ${ }^{4,7}$ Studies investigating the inflammatory effects of PEMs have reported significant alteration in macrophage adhesion, monocyte/macrophage differentiation, the secretion of inflammatory cytokines, and the formation of foreign body giant cells, depending on the respective assembling solutions used as PEM substrates. ${ }^{55,56}$ Testing of three PEM coatings modified in a gradient fashion with respect to wettability in our study revealed distinct levels of cellular activation and cytokine release by PBMCs in response to these coatings. In accordance with previous findings on other substrates, ${ }^{8,9}$ reduced surface wettability on the PEM coating cationic A led to a lower immune response, while the inflammatory response was enhanced in response to the more hydrophobic coating cationic $\mathrm{C}$. This knowledge could be used for the modification of existing biomaterials and medical devices so that desired immune responses for any given biomaterial could be imparted to existing devices with the application of the appropriate PEM coating. Due to the low thickness of the PEMs in the nanometer range, the surface topography of the underlying materials can be preserved while physicochemical properties such as wettability can be modified in the desired way by the selected conditions in the coating process. ${ }^{57}$ Thus, PEM coatings could be a versatile tool to maintain the functionality of a medical device while meliorating immune profiles and subsequently improving implant biocompatibility and longevity.

The distinct immune responses provoked by the PEM coatings cationic $\mathrm{A}$ and $\mathrm{C}$ were examined in detail in this study. The immune response observed by three donors was found to be broadly reflected by a larger cohort of 24 donors (Figure 4). Although the monocyte response showed a degree of variation across individuals, as expected, the overall trends for the different PEM coatings were the same for the vast majority of the 24 donors tested. Thus, the analysis of a small number of PBMC donors tested in this study seems to be representative of a larger cohort. Nevertheless, testing a large number of donors naturally increases the statistical power of the analysis, which is apparent for IL-8 in our experiments (testing three donors showed no significant difference between cationic A and C (Figure 3B), while analysis of 24 donors showed a significant increase in the IL-8 expression for cationic C ( $p=$ 0.0042 , Figure $4 \mathrm{~A})$ ). In contrast to prior studies, ${ }^{58,59}$ no gender-specific differences were discovered and only a very 
weak trend toward a modest rise in TNF- $\alpha$ expression with increasing donor age was observed. These findings suggest that demographic differences in the selection of PBMC donors are not factors of primary importance and indicate that the personalized selection of implant materials may only be necessary for the most extreme outliers in biological response. However, other factors such as environmental differences, the presence of infections, hypersensitivity, or allergies, or even activation of the immune systems in response to previously implanted biomaterials might affect the immune response but were not examined in this study.

We observed the distinct cellular response to coatings $\mathrm{A}$ and $\mathrm{C}$ to be only apparent in the presence of human serum (Figure 5 ). In contrast to the immune response in serum-containing cultures, cytokine release under serum-free conditions was significantly increased and no differences between the PEM coatings were discernible. By precoating the surfaces with serum, we found the difference in the inflammatory response between surfaces cationic $\mathrm{A}$ and $\mathrm{C}$ to be restored, even in cultures using serum-free media. This is in line with previous studies $^{60}$ and clearly indicates a decisive role of the adsorbed protein layer in the observed immune response toward PEM coatings. Upon implantation, biomaterials are covered by a layer of proteins within seconds. ${ }^{10}$ Our results show that these proteins are the driving force and determine the subsequent immunological response to PEMs, while biomaterial surface properties appear to primarily influence the composition of the adsorbed proteins rather than influencing the immune response.

Examining the proteins adsorbed onto the PEMs investigated in this study identified 26 proteins on the surface of the coatings, while the total amount of proteins on the more hydrophobic cationic $\mathrm{C}$ surface was 1.76-fold higher than on the hydrophilic cationic A, thus indicating a wettabilityassociated effect. This is in line with previous findings showing that proteins bind to hydrophilic surfaces to a lower extent and less tightly than to hydrophobic surfaces. ${ }^{8}$ All surfaces investigated here showed high abundance and number of apolipoproteins. The high affinity of apolipoproteins to biomaterial surfaces has already been reported in the literature. $^{14,61,62}$ Of note is that the adsorption of apolipoproteins onto the PEM surfaces studied here exceeded the extent of adsorption to most other materials described so far. While apolipoproteins were originally mainly associated with their function as lipid transporters in high-density lipoproteins (HDLs), very low density lipoproteins (VLDLs), or lowdensity lipoproteins (LDLs), there is now increasing evidence that they are also involved in the modulation of immunological processes. Apolipoprotein A-II is associated with inflammation, ${ }^{46}$ enhances proinflammatory monocyte responses to LPS, ${ }^{47}$ and correlates to monocyte activation in sepsis patients. $^{48}$ Apolipoprotein C-III was shown to significantly increase TNF- $\alpha$, IL-6, IL- $1 \beta$, and MCP-1 release from monocytes ${ }^{49,50}$ while apolipoprotein $\mathrm{J} /$ Clusterin was found to induce matrix metalloproteinase- 9 expression in monocytes. $^{63}$ The proinflammatory characteristic of these apolipoproteins was also shared by other identified proteins HRG and SAA4, which were shown to mediate the transition of alternatively activated (M2) to proinflammatory (M1) macrophages in a tumor model ${ }^{64}$ and induce expression of proinflammatory cytokines TNF- $\alpha$, IL- $1 \beta$, and IL- 8 in neutrophils and monocytes, ${ }^{44,45}$ respectively. Together, these proteins with inflammatory function or being associated with proinflammatory effects based on the literature (HRG, SAA4, and apolipoproteins A-II, C-III, J/Clusterin) showed an abundance of $34.9 \%$ on the more proinflammatory cationic C surface, while these proteins only accounted for $12.2 \%$ of proteins on the less inflammatory cationic A coating (2.9-fold increase). When looking at the absolute amount of protein on the surfaces, this effect becomes even more pronounced. Due to the 1.76-fold increased total protein amount on surface cationic $\mathrm{C}, 5$ times the amount of proinflammatory proteins is found on this surface in comparison to cationic $\mathrm{A}$ in absolute terms. In contrast, other apolipoproteins are associated with anti-inflammatory effects. Apolipoproteins A-I and E inhibit the production of proinflammatory cytokines and promote macrophage conversion from the proinflammatory M1 to the anti-inflammatory M2 phenotype. ${ }^{51,65,66}$ These two inflammation-dampening proteins accounted for $53.6 \%$ of the total protein abundance on cationic $\mathrm{A}$, while their abundance on cationic $\mathrm{C}$ was only $23.6 \%$. Thus, the abundance of proteins with inflammation-dampening properties on the more inflammatory cationic $\mathrm{C}$ surface was less than half than those on the less inflammatory PEM cationic A. In addition to protein identity, wettability-dependent conformational changes and/or protein unfolding can further enhance the observed effect. ${ }^{67}$ Hereby, nonpolar hydrophobic surfaces seem to cause the most protein unfolding, while neutrally charged hydrophilic surfaces tend to induce the least changes in the protein structure. ${ }^{68}$ Unfolding of proteins can influence monocytes by regulating the expression of proinflammatory cytokines and modulating cell attachment. ${ }^{69}$ Thus, these effects might be additional factors further enhancing the immune response for the more hydrophobic PEM coating cationic C. Taken together, protein analysis shows that the composition of the adsorbed protein layer can have a major impact on the cytokine expression by monocytes and the resulting immune response. In this context, the class of apolipoproteins comes into focus as a promising candidate to modulate the immune response to biomaterials.

To gain a better understanding of how the adsorbed proteins influence the observed immune responses, an analysis of intracellular signaling was performed to provide information about the type of cellular activation. We found an elevated expression of c-Jun and HSP70 in response to surface cationic $\mathrm{C}$, and higher expression of MEK $1+2, \mathrm{NF}-\kappa \mathrm{B}$, p-Erk1, and TRAF1 to surface cationic A (Figure 7). Most of these proteins are assigned to MAPK signaling, a cascade that is well established for its role in the regulation of the inflammatory response in innate immune cells. ${ }^{70}$ As such, the production of several proinflammatory cytokines like TNF- $\alpha$, IL- 6 , or IL- $1 \beta$ as well as other inflammatory mediators are positively regulated by MAPK. While the upregulation of c-Jun, an important regulator of gene expression, on the cationic $\mathrm{C}$ coating is in line with the increased cytokine response to this surface, the elevated expression of other proteins of the classical MAPK pathway in response to the cationic A surface suggests that these proteins do not play a major role in mediating inflammatory responses to the coatings investigated in this study. Large pathways like MAPK also have a high level of complexity and can be modulated by various mechanisms at many steps of the pathway. As a result, differences for individual members may develop. In addition, protein conditions like phosphorylation can occur dynamically, while the measured cytokines accumulated over time. It should also be pointed out that cytokine response, in general, is a signal 
that may undergo several steps of amplification and thus may be significantly lower at the signaling pathway level investigated here. A single-cell analysis technique would shed more light on the possible heterogeneity of responses as the results observed may have been partially masked by a fraction of cells responding in a different manner (for example, the results in Figure 3 showed a range of $25-90 \%$ of nonpositive monocytes, depending on the respective cytokines). However, the identification of multiple proteins from the MAPK pathway implicates a role for this signaling route as a mechanism that monocytes used to respond to differences in the two PEM coatings investigated.

\section{CONCLUSIONS}

In this study, PEM coatings were developed for the targeted modification of surface properties including wettability. We demonstrated coating-dependent adsorption of surface proteins, resulting in distinct degrees of inflammation. These effects were accompanied by differences in intracellular signaling molecules. This study thus demonstrates the interrelation of material properties, protein adsorption, and the immune response as closely connected aspects in the biological response to synthetic materials.

\section{ASSOCIATED CONTENT}

\section{SI Supporting Information}

The Supporting Information is available free of charge at https://pubs.acs.org/doi/10.1021/acsami.1c16175.

Gating strategy used to analyze surface marker proteins on PBMCs; gating strategy used for intracellular cytokine staining analysis; cytokine secretion of PBMCs cultured on PEM surfaces; intracellular cytokine analysis across time of PBMCs cultured on PEM coatings; age-associated intracellular cytokine expression in monocytes in a cohort of 24 individuals; intracellular signaling molecules in monocytes with significant differences in expression between negative and positive controls; sex, age, and frequency of major cellular populations in PBMC; abundance (percentage) of proteins identified on cationic $\mathrm{A}$, cationic $\mathrm{C}$, and the negative control (TCP) using LC-MS; statistical analysis comparing the amount of proteins on the cationic $\mathrm{A}$ and cationic C PEM surfaces; and expression levels of intracellular proteins analyzed with DigiWest technology (PDF)

\section{AUTHOR INFORMATION}

\section{Corresponding Author}

Florian Billing - NMI Natural and Medical Sciences Institute at the University of Tübingen, 72770 Reutlingen, Germany; () orcid.org/0000-0002-3874-9012; Phone: +49 7121 51530843; Email: florian.billing@nmi.de

\section{Authors}

Bernadette Walter - NMI Natural and Medical Sciences Institute at the University of Tübingen, 72770 Reutlingen, Germany

Simon Fink - NMI Natural and Medical Sciences Institute at the University of Tübingen, 72770 Reutlingen, Germany

Elsa Arefaine - NMI Natural and Medical Sciences Institute at the University of Tübingen, 72770 Reutlingen, Germany
Luisa Pickarski - NMI Natural and Medical Sciences Institute at the University of Tübingen, 72770 Reutlingen, Germany

Sandra Maier - NMI Natural and Medical Sciences Institute at the University of Tübingen, 72770 Reutlingen, Germany

Robin Kretz - NMI Natural and Medical Sciences Institute at the University of Tübingen, 72770 Reutlingen, Germany

Meike Jakobi - NMI Natural and Medical Sciences Institute at the University of Tübingen, 72770 Reutlingen, Germany

Nora Feuerer - NMI Natural and Medical Sciences Institute at the University of Tübingen, 72770 Reutlingen, Germany; Department of Biomedical Engineering, Eberhard Karls University Tübingen, 72076 Tübingen, Germany

Nicole Schneiderhan-Marra - NMI Natural and Medical Sciences Institute at the University of Tübingen, 72770 Reutlingen, Germany

Claus Burkhardt - NMI Natural and Medical Sciences Institute at the University of Tübingen, 72770 Reutlingen, Germany

Markus Templin - NMI Natural and Medical Sciences Institute at the University of Tübingen, 72770 Reutlingen, Germany

Anne Zeck - NMI Natural and Medical Sciences Institute at the University of Tübingen, 72770 Reutlingen, Germany

Rumen Krastev - NMI Natural and Medical Sciences Institute at the University of Tübingen, 72770 Reutlingen, Germany; Faculty of Applied Chemistry, Reutlingen University, 72762 Reutlingen, Germany

Hanna Hartmann - NMI Natural and Medical Sciences Institute at the University of Tübingen, 72770 Reutlingen, Germany

Christopher Shipp - NMI Natural and Medical Sciences Institute at the University of Tübingen, 72770 Reutlingen, Germany

Complete contact information is available at: https://pubs.acs.org/10.1021/acsami.1c16175

\section{Notes}

The authors declare no competing financial interest.

\section{ACKNOWLEDGMENTS}

This work received financial support from the EU-EFRE and the State of Baden-Wuerttemberg (712889) as well as by the State Ministry of Baden-Wuerttemberg for Economic Affairs, Labour and Tourism. We are very grateful to Burkhard Schlosshauer (NMI) for his contribution to the initiation of the project. Further thanks go to Laura Strano (NMI) for her essential contribution to the PEMs tested here, Alexander Rudt for helpful discussions, and Hannah Graf for her assistance in the SEM analysis. The table of contents illustration was created using biorender.com.

\section{REFERENCES}

(1) Anderson, J. M. Biological Responses to Materials. Annu. Rev. Mater. Res. 2001, 31, 81-110.

(2) Anderson, J. M.; Rodriguez, A.; Chang, D. T. Foreign Body Reaction to Biomaterials. Semin. Immunol. 2008, 20, 86-100.

(3) Williams, D. F. On the Mechanisms of Biocompatibility. Biomaterials 2008, 29, 2941-2953.

(4) Franz, S.; Rammelt, S.; Scharnweber, D.; Simon, J. C. Immune responses to implants - a review of the implications for the design of immunomodulatory biomaterials. Biomaterials 2011, 32, 6692-6709.

(5) Rostam, H. M.; Singh, S.; Vrana, N. E.; Alexander, M. R.; Ghaemmaghami, A. M. Impact of surface chemistry and topography 
on the function of antigen presenting cells. Biomater. Sci. 2015, 3, 424-441.

(6) Albanese, A.; Tang, P. S.; Chan, W. C. The effect of nanoparticle size, shape, and surface chemistry on biological systems. Annu. Rev. Biomed. Eng. 2012, 14, 1-16.

(7) Mariani, E.; Lisignoli, G.; Borzì, R. M.; Pulsatelli, L. Biomaterials: Foreign Bodies or Tuners for the Immune Response? Int. J. Mol. Sci. 2019, 20, No. 636.

(8) Thevenot, P.; Hu, W.; Tang, L. Surface chemistry influences implant biocompatibility. Curr. Top. Med. Chem. 2008, 8, 270-280.

(9) Brodbeck, W. G.; Voskerician, G.; Ziats, N. P.; Nakayama, Y.; Matsuda, T.; Anderson, J. M. In vivo leukocyte cytokine mRNA responses to biomaterials are dependent on surface chemistry. $J$. Biomed. Mater. Res., Part A 2003, 64A, 320-329.

(10) Horbett, T. A. Chapter 13 Principles underlying the role of adsorbed plasma proteins in blood interactions with foreign materials. Cardiovasc. Pathol. 1993, 2, 137-148.

(11) Wilson, C. J.; Clegg, R. E.; Leavesley, D. I.; Pearcy, M. J. Mediation of biomaterial-cell interactions by adsorbed proteins: a review. Tissue Eng. 2005, 11, 1-18.

(12) Jenney, C. R.; Anderson, J. M. Adsorbed serum proteins responsible for surface dependent human macrophage behavior. $J$. Biomed. Mater. Res. 2000, 49, 435-447.

(13) Collier, T. O.; Anderson, J. M. Protein and surface effects on monocyte and macrophage adhesion, maturation, and survival. $J$. Biomed. Mater. Res. 2002, 60, 487-496.

(14) Visalakshan, R. M.; MacGregor, M. N.; Sasidharan, S.; Ghazaryan, A.; Mierczynska-Vasilev, A. M.; Morsbach, S.; Mailänder, V.; Landfester, K.; Hayball, J. D.; Vasilev, K. Biomaterial Surface Hydrophobicity-Mediated Serum Protein Adsorption and Immune Responses. ACS Appl. Mater. Interfaces 2019, 11 (), 2761527623 DOI: 10.1021 /acsami.9b09900.

(15) Kim, Y. K.; Que, R.; Wang, S.-W.; Liu, W. F. Modification of biomaterials with a self-protein inhibits the macrophage response. Adv. Healthcare Mater. 2014, 3 (), 989-994 DOI: 10.1002/ adhm.201300532.

(16) Roach, P.; Eglin, D.; Rohde, K.; Perry, C. C. Modern biomaterials: a review-bulk properties and implications of surface modifications. J. Mater. Sci.: Mater. Med. 2007, 18, 1263-1277.

(17) Sigal, G. B.; Mrksich, M.; Whitesides, G. M. Effect of Surface Wettability on the Adsorption of Proteins and Detergents. J. Am. Chem. Soc. 1998, 120, 3464-3473.

(18) Kang, C.-K.; Lee, Y.-S. The surface modification of stainless steel and the correlation between the surface properties and protein adsorption. J. Mater. Sci.: Mater. Med. 2007, 18 (), 1389-1398 DOI: $10.1007 / \mathrm{s} 10856-006-0079-9$.

(19) Brodbeck, W. G.; Colton, E.; Anderson, J. M. Effects of adsorbed heat labile serum proteins and fibrinogen on adhesion and apoptosis of monocytes/macrophages on biomaterials. J. Mater. Sci.: Mater. Med. 2003, 14, 671-675.

(20) Decher, G.; Hong, J. D.; Schmitt, J. Buildup of ultrathin multilayer films by a self-assembly process: III. Consecutively alternating adsorption of anionic and cationic polyelectrolytes on charged surfaces. Thin Solid Films 1992, 210-211, 831-835.

(21) Detzel, C. J.; Larkin, A. L.; Rajagopalan, P. Polyelectrolyte multilayers in tissue engineering. Tissue Eng., Part B 2011, 17, 101.

(22) Yoo, D.; Shiratori, S. S.; Rubner, M. F. Controlling Bilayer Composition and Surface Wettability of Sequentially Adsorbed Multilayers of Weak Polyelectrolytes. Macromolecules 1998, 31, 4309-4318.

(23) Krastev, R.; Rudt, A.; Xiong, X.; Hartmann, H. Polyelectrolyte Coatings for Surface Modification of Medical Implants. Curr. Dir. Biomed. Eng. 2018, 4, 217-220.

(24) Trindade, M. In vitro reaction to orthopaedic biomaterials by macrophages and lymphocytes isolated from patients undergoing revision surgery. Biomaterials 2001, 22, 253-259.

(25) Brodbeck, W. G.; MacEwan, M.; Colton, E.; Meyerson, H.; Anderson, J. M. Lymphocytes and the foreign body response:
Lymphocyte enhancement of macrophage adhesion and fusion. J. Biomed. Mater. Res., Part A 2005, 74A, 222-229.

(26) Jenney, C. R.; Anderson, J. M. Adsorbed IgG: A potent adhesive substrate for human macrophages. J. Biomed. Mater. Res. 2000, 50, 281-290.

(27) Maciel, J.; Oliveira, M. I.; Gonçalves, R. M.; Barbosa, M. A. The effect of adsorbed fibronectin and osteopontin on macrophage adhesion and morphology on hydrophilic and hydrophobic model surfaces. Acta Biomater. 2012, 8 (), 3669-3677 DOI: 10.1016/ j.actbio.2012.06.010.

(28) Park, K.; Shim, H. S.; Dewanjee, M. K.; Eigler, N. L. In vitro and in vivo studies of PEO-grafted blood-contacting cardiovascular prostheses. J. Biomater. Sci., Polym. Ed. 2000, 11, 1121-1134.

(29) Ngo, B. K. D.; Grunlan, M. A. Protein Resistant Polymeric Biomaterials. ACS Macro Lett. 2017, 6, 992-1000.

(30) Friedrichs, B. Th. Peters. Jr.: All about Albumin. Biochemistry, Genetics, and Medical Applications. XX and 432 pages, numerous figures and tables. Academic Press, Inc., San Diego, California, 1996. Price: 85.00 US \$. Food/Nahrung 1997, 41, 382.

(31) Oyane, A.; Kim, H.-M.; Furuya, T.; Kokubo, T.; Miyazaki, T.; Nakamura, T. Preparation and assessment of revised simulated body fluids. J. Biomed. Mater. Res. 2003, 65A, 188-195.

(32) Billing, F.; Jakobi, M.; Martin, D.; Gerlach, K.; Arefaine, E.; Weiss, M.; Schneiderhan-Marra, N.; Hartmann, H.; Shipp, C. The immune response to the SLActive titanium dental implant surface in vitro is predominantly driven by innate immune cells. J. Immunol. Regener. Med. 2021, 13, No. 100047.

(33) Segan, S.; Jakobi, M.; Khokhani, P.; Klimosch, S.; Billing, F.; Schneider, M.; Martin, D.; Metzger, U.; Biesemeier, A.; Xiong, X.; Mukherjee, A.; Steuer, H.; Keller, B. M.; Joos, T.; Schmolz, M.; Rothbauer, U.; Hartmann, H.; Burkhardt, C.; Lorenz, G.; Schneiderhan-Marra, N.; Shipp, C. Systematic Investigation of Polyurethane Biomaterial Surface Roughness on Human Immune Responses in vitro. BioMed Res. Int. 2020, 2020, No. 3481549.

(34) European Medicines Agency. Guideline on Bioanalytical Method Validation; European Medicines Agency, 2011. https://www.ema. europa.eu/en/documents/scientific-guideline/guideline-bioanalyticalmethod-validation en.pdf (accessed Feb 16, 2021).

(35) U.S. Department of Health and Human Services, Food and Drug Administration. Bioanalytical Method Validation:Guidance for Industry; U.S. Department of Health and Human Services, Food and Drug Administration, 2018. https://www.fda.gov/downloads/drugs/ guidances/ucm070107.Pdf (accessed Feb 11, 2021).

(36) Westgard, J. O.; Barry, P. L.; Hunt, M. R.; Groth, T. A multirule Shewhart chart for quality control in clinical chemistry. Clin. Chem. 1981, 27, 493-501.

(37) Cox, J.; Mann, M. MaxQuant enables high peptide identification rates, individualized p.p.b.-range mass accuracies and proteome-wide protein quantification. Nat. Biotechnol. 2008, 26, $1367-1372$.

(38) Tyanova, S.; Temu, T.; Sinitcyn, P.; Carlson, A.; Hein, M. Y.; Geiger, T.; Mann, M.; Cox, J. The Perseus computational platform for comprehensive analysis of (prote)omics data. Nat. Methods 2016, 13, $731-740$

(39) Schwanhäusser, B.; Busse, D.; Li, N.; Dittmar, G.; Schuchhardt, J.; Wolf, J.; Chen, W.; Selbach, M. Global quantification of mammalian gene expression control. Nature 2011, 473, 337-342.

(40) Treindl, F.; Ruprecht, B.; Beiter, Y.; Schultz, S.; Döttinger, A.; Staebler, A.; Joos, T. O.; Kling, S.; Poetz, O.; Fehm, T.; Neubauer, H.; Kuster, B.; Templin, M. F. A bead-based western for high-throughput cellular signal transduction analyses. Nat. Commun. 2016, 7, No. 12852.

(41) Saeed, A. I.; Bhagabati, N. K.; Braisted, J. C.; Liang, W.; Sharov, V.; Howe, E. A.; Li, J.; Thiagarajan, M.; White, J. A.; Quackenbush, J. [9] TM4 Microarray Software Suite. In Methods in Enzymology; Kimmel, A. R.; Oliver, B., Eds.; DNA Microarrays, Part B: Databases and Statistics; Elsevier/Academic Press, 2006; Vol. 411, pp 134-193.

(42) Tak, T.; van Groenendael, R.; Pickkers, P.; Koenderman, L. Monocyte Subsets Are Differentially Lost from the Circulation during 
Acute Inflammation Induced by Human Experimental Endotoxemia.

J. Innate Immun. 2017, 9 (), 464-474 DOI: 10.1159/000475665.

(43) Trinschek, B.; Lüssi, F.; Haas, J.; Wildemann, B.; Zipp, F.; Wiendl, H.; Becker, C.; Jonuleit, H. Kinetics of IL-6 Production Defines $\mathrm{T}$ Effector Cell Responsiveness to Regulatory $\mathrm{T}$ Cells in Multiple Sclerosis. PLoS One 2013, 8, No. e77634.

(44) Furlaneto, C. J.; Campa, A. A novel function of serum amyloid A: a potent stimulus for the release of tumor necrosis factor-alpha, interleukin-1beta, and interleukin- 8 by human blood neutrophil. Biochem. Biophys. Res. Commun. 2000, 268, 405.

(45) Ribeiro, F. P.; Furlaneto, C. J.; Hatanaka, E.; Ribeiro, W. B.; Souza, G. M.; Cassatella, M. A.; Campa, A. mRNA expression and release of interleukin- 8 induced by serum amyloid A in neutrophils and monocytes. Mediators Inflammation 2003, 12, 173-178.

(46) Yang, M.; Liu, Y.; Dai, J.; Li, L.; Ding, X.; Xu, Z.; Mori, M.; Miyahara, H.; Sawashita, J.; Higuchi, K. Apolipoprotein A-II induces acute-phase response associated AA amyloidosis in mice through conformational changes of plasma lipoprotein structure. Sci. Rep. 2018, 8, No. 5620.

(47) Thompson, P. A.; Berbée, J. F.; Rensen, P. C.; Kitchens, R. L. Apolipoprotein A-II augments monocyte responses to LPS by suppressing the inhibitory activity of LPS-binding protein. Innate Immun. 2008, 14, 365.

(48) Barlage, S.; Gnewuch, C.; Liebisch, G.; Wolf, Z.; Audebert, F.X.; Glück, T.; Fröhlich, D.; Krämer, B. K.; Rothe, G.; Schmitz, G. Changes in HDL-associated apolipoproteins relate to mortality in human sepsis and correlate to monocyte and platelet activation. Intensive Care Med. 2009, 35, 1877-1885.

(49) Zewinger, S.; Reiser, J.; Jankowski, V.; Alansary, D.; Hahm, E.; Triem, S.; Klug, M.; Schunk, S. J.; Schmit, D.; Kramann, R.; Körbel, C.; Ampofo, E.; Laschke, M. W.; Selejan, S.-R.; Paschen, A.; Herter, T.; Schuster, S.; Silbernagel, G.; Sester, M.; Sester, U.; Aßmann, G.; Bals, R.; Kostner, G.; Jahnen-Dechent, W.; Menger, M. D.; Rohrer, L.; März, W.; Böhm, M.; Jankowski, J.; Kopf, M.; Latz, E.; Niemeyer, B. A.; Fliser, D.; Laufs, U.; Speer, T. Apolipoprotein C3 induces inflammation and organ damage by alternative inflammasome activation. Nat. Immunol. 2020, 21, 30-41.

(50) Han, X.; Wang, T.; Zhang, J.; Liu, X.; Li, Z.; Wang, G.; Song, Q.; Pang, D.; Ouyang, H.; Tang, X. Apolipoprotein CIII regulates lipoprotein-associated phospholipase A2 expression via the MAPK and NF $\kappa$ B pathways. Biol. Open 2015, 4, 661-665.

(51) Hyka, N.; Dayer, J. M.; Modoux, C.; Kohno, T.; Edwards, C. K.; Roux-Lombard, P.; Burger, D. Apolipoprotein A-I inhibits the production of interleukin-1beta and tumor necrosis factor-alpha by blocking contact-mediated activation of monocytes by $\mathrm{T}$ lymphocytes. Blood 2001, 97, 2381-2389.

(52) Lee, S. H.; Soyoola, E.; Chanmugam, P.; Hart, S.; Sun, W.; Zhong, H.; Liou, S.; Simmons, D.; Hwang, D. Selective expression of mitogen-inducible cyclooxygenase in macrophages stimulated with lipopolysaccharide. J. Biol. Chem. 1992, 267, 25934-25938.

(53) Bode, J. G.; Ehlting, C.; Häussinger, D. The macrophage response towards LPS and its control through the p38MAPK-STAT3 axis. Cell. Signalling 2012, 24, 1185-1194.

(54) Liu, X.; Yin, S.; Chen, Y.; Wu, Y.; Zheng, W.; Dong, H.; Bai, Y.; Qin, Y.; Li, J.; Feng, S.; Zhao, P. LPS-induced proinflammatory cytokine expression in human airway epithelial cells and macrophages via NF- $\kappa$ B, STAT3 or AP-1 activation. Mol. Med. Rep. 2018, 17, 5484-5491.

(55) Zhou, G.; Niepel, M. S.; Saretia, S.; Groth, T. Reducing the inflammatory responses of biomaterials by surface modification with glycosaminoglycan multilayers. J. Biomed. Mater. Res. 2016, 104, 493502.

(56) Knopf-Marques, H.; Singh, S.; Htwe, S. S.; Wolfova, L.; Buffa, R.; Bacharouche, J.; Francius, G.; Voegel, J.-C.; Schaaf, P.; Ghaemmaghami, A. M.; Vrana, N. E.; Lavalle, P. Immunomodulation with Self-Crosslinked Polyelectrolyte Multilayer-Based Coatings. Biomacromolecules 2016, 17, 2189-2198.
(57) Hartmann, H.; Krastev, R. Biofunctionalization of surfaces using polyelectrolyte multilayers. BioNanoMaterials 2017, 18, No. 20160015.

(58) Bernin, H.; Fehling, H.; Marggraff, C.; Tannich, E.; Lotter, H. The cytokine profile of human NKT cells and PBMCs is dependent on donor sex and stimulus. Med. Microbiol. Immunol. 2016, 205 (), 321-332 DOI: 10.1007/s00430-016-0449-y.

(59) Longo, D. M.; Louie, B.; Putta, S.; Evensen, E.; Ptacek, J.; Cordeiro, J.; Wang, E.; Pos, Z.; Hawtin, R. E.; Marincola, F. M.; Cesano, A. Single-cell network profiling of peripheral blood mononuclear cells from healthy donors reveals age- and raceassociated differences in immune signaling pathway activation. $J$. Immunol. 2012, 188 (), 1717-1725 DOI: 10.4049/jimmunol.1102514.

(60) Allen, L. T.; Tosetto, M.; Miller, I. S.; O’Connor, D. P.; Penney, S. C.; Lynch, I.; Keenan, A. K.; Pennington, S. R.; Dawson, K. A.; Gallagher, W. M. Surface-induced changes in protein adsorption and implications for cellular phenotypic responses to surface interaction. Biomaterials 2006, 27, 3096-3108.

(61) Magnani, A.; Barbucci, R.; Lamponi, S.; Chiumento, A.; Paffetti, A.; Trabalzini, L.; Martelli, P.; Santucci, A. Two-step elution of human serum proteins from different glass-modified bioactive surfaces: a comparative proteomic analysis of adsorption patterns. Electrophoresis 2004, 25, 2413.

(62) Cornelius, R. M.; Archambault, J.; Brash, J. L. Identification of apolipoprotein A-I as a major adsorbate on biomaterial surfaces after blood or plasma contact. Biomaterials 2002, 23, 3583-3587.

(63) Shim, Y.-J.; Kang, B.-H.; Jeon, H.-S.; Park, I.-S.; Lee, K.-U.; Lee, I.-K.; Park, G.-H.; Lee, K.-M.; Schedin, P.; Min, B.-H. Clusterin induces matrix metalloproteinase- 9 expression via ERK1/2 and PI3K/ Akt/NF- $\kappa \mathrm{B}$ pathways in monocytes/macrophages. J. Leukocyte Biol. 2011, 90, 761-769.

(64) Rolny, C.; Mazzone, M.; Tugues, S.; Laoui, D.; Johansson, I.; Coulon, C.; Squadrito, M. L.; Segura, I.; Li, X.; Knevels, E.; Costa, S.; Vinckier, S.; Dresselaer, T.; Åkerud, P.; De, M. M.; Salomäki, H.; Phillipson, M.; Wyns, S.; Larsson, E.; Buysschaert, I.; Botling, J.; Himmelreich, U.; Van, G. J. A.; De, P. M.; Dewerchin, M.; ClaessonWelsh, L.; Carmeliet, P. HRG inhibits tumor growth and metastasis by inducing macrophage polarization and vessel normalization through downregulation of PIGF. Cancer Cell 2011, 19, 31.

(65) Baitsch, D.; Bock, H. H.; Engel, T.; Telgmann, R.; MüllerTidow, C.; Varga, G.; Bot, M.; Herz, J.; Robenek, H.; von, E. A.; Nofer, J. R. Apolipoprotein E induces antiinflammatory phenotype in macrophages. Arterioscler., Thromb., Vasc. Biol. 2011, 31, 1160.

(66) Murphy, A. J.; Woollard, K. J.; Hoang, A.; Mukhamedova, N.; Stirzaker, R. A.; McCormick, S. P.; Remaley, A. T.; Sviridov, D.; ChinDusting, J. High-density lipoprotein reduces the human monocyte inflammatory response. Arterioscler., Thromb., Vasc. Biol. 2008, 28, 2071.

(67) Gittens, R. A.; Scheideler, L.; Rupp, F.; Hyzy, S. L.; GeisGerstorfer, J.; Schwartz, Z.; Boyan, B. D. A review on the wettability of dental implant surfaces II: Biological and clinical aspects. Acta Biomater. 2014, 10, 2907-2918.

(68) Brash, J. L.; Horbett, T. A.; Latour, R. A.; Tengvall, P. The blood compatibility challenge. Part 2: Protein adsorption phenomena governing blood reactivity. Acta Biomater. 2019, 94, 11-24.

(69) Visalakshan, R. M.; Cavallaro, A. A.; MacGregor, M. N.; Lawrence, E. P.; Koynov, K.; Hayball, J. D.; Vasilev, K. Nanotopography-Induced Unfolding of Fibrinogen Modulates Leukocyte Binding and Activation. Adv. Funct. Mater. 2019, 29, No. 1807453.

(70) Arthur, J. S. C.; Ley, S. C. Mitogen-activated protein kinases in innate immunity. Nat. Rev. Immunol. 2013, 13 (), 679-692 DOI: $10.1038 /$ nri3495. 